\title{
How Local Excitation-Inhibition Ratio Impacts the Whole Brain Dynamics
}

\author{
Gustavo Deco, ${ }^{1,2 \star}$ Adrián Ponce-Alvarez, ${ }^{1 \star}$ Patric Hagmann, ${ }^{3,4}$ Gian Luca Romani, ${ }^{5}$ Dante Mantini, ${ }^{6,7}$ \\ and Maurizio Corbetta ${ }^{8}$ \\ ${ }^{1}$ Center for Brain and Cognition, Computational Neuroscience Group, Department of Information and Communication Technologies, and ${ }^{2}$ Institució \\ Catalana de la Recerca i Estudis Avançats (ICREA), Universitat Pompeu Fabra, Barcelona, 08010, Spain, ${ }^{3}$ Department of Radiology, Lausanne University \\ Hospital and University of Lausanne (CHUV-UNIL), 1011 Lausanne, Switzerland, ${ }^{4}$ Signal Processing Laboratory 5, Ecole Polytechnique Fédérale de \\ Lausanne (EPFL), 1015 Lausanne, Switzerland, ${ }^{5}$ Institute of Advanced Biomedical Technologies, G. d'Annunzio University Foundation, Department of \\ Neuroscience and Imaging, G. d'Annunzio University, Chieti, 66013, Italy, ${ }^{6}$ Department of Health Sciences and Technology, ETH Zurich, 8057, Zurich, \\ Switzerland, ${ }^{7}$ Department of Experimental Psychology, University of Oxford, OX1 3UD, Oxford, United Kingdom, and ${ }^{8}$ Department of Neurology, \\ Radiology, Anatomy of Neurobiology, School of Medicine, Washington University in St Louis, St Louis, Missouri 63110
}

The spontaneous activity of the brain shows different features at different scales. On one hand, neuroimaging studies show that longrange correlations are highly structured in spatiotemporal patterns, known as resting-state networks, on the other hand, neurophysiological reports show that short-range correlations between neighboring neurons are low, despite a large amount of shared presynaptic inputs. Different dynamical mechanisms of local decorrelation have been proposed, among which is feedback inhibition. Here, we investigated the effect of locally regulating the feedback inhibition on the global dynamics of a large-scale brain model, in which the long-range connections are given by diffusion imaging data of human subjects. We used simulations and analytical methods to show that locally constraining the feedback inhibition to compensate for the excess of long-range excitatory connectivity, to preserve the asynchronous state, crucially changes the characteristics of the emergent resting and evoked activity. First, it significantly improves the model's prediction of the empirical human functional connectivity. Second, relaxing this constraint leads to an unrealistic network evoked activity, with systematic coactivation of cortical areas which are components of the default-mode network, whereas regulation of feedback inhibition prevents this. Finally, information theoretic analysis shows that regulation of the local feedback inhibition increases both the entropy and the Fisher information of the network evoked responses. Hence, it enhances the information capacity and the discrimination accuracy of the global network. In conclusion, the local excitation-inhibition ratio impacts the structure of the spontaneous activity and the information transmission at the large-scale brain level.

Key words: anatomical connectivity; functional connectivity; large-scale brain model; local feedback inhibition; resting-state activity

\section{Introduction}

The spontaneous activity of the brain, i.e., not stimulus- or taskdriven, shows different features at different spatial scales. Indeed, although long-range activity correlations between brain regions are highly and strongly structured in spatiotemporal patterns, known as resting-state networks, on one hand, short-range cor-

Received Dec. 4, 2013; revised April 25, 2014; accepted April 30, 2014.

Author contributions: G.D. and A.P.-A. designed research; G.D. and A.P.-A. performed research; G.D., A.P.-A., P.H., G.L.R., D.M., and M.C. analyzed data; G.D. and A.P.-A. wrote the paper.

G.D. was supported by the ERC Advanced Grant: DYSTRUCTURE (no. 295129), by the Spanish Research Project SAF2010-16085 and by the CONSOLIDER-INGENI0 2010 Program CSD2007-00012, the Foundation "La Marato" (Catalonia), and the FP7-ICT BrainScales and The Human Brain Project FET-Flagship. P.H was supported by Leenaards Foundation. M.C. was supported by NIH Grants R01HD061117 and R01MH096482. The research reported herein was supported by the Brain Network Recovery Group through the James S. McDonnell Foundation. We thank H. Sompolinsky and M. Lechón for critical comments and valuable discussions.

The authors declare no competing financial interests.

*G.D. and A.P.-A. contributed equally to this work.

Correspondence should be addressed to Dr Adrián Ponce-Alvarez, Universitat Pompeu Fabra C/ Roc Boronat, 138, 08018 Barcelona, Spain. E-mail: adrian.ponce@upf.edu.

DOI:10.1523/JNEUROSCI.5068-13.2014

Copyright $\odot 2014$ the authors $\quad 0270-6474 / 14 / 347886-13 \$ 15.00 / 0$ relations within local circuits are low or even negligible, on the other hand.

Numerous neuroimaging experiments demonstrate the existence of spontaneous long-range correlations, i.e., resting functional connectivity (FC), by fMRI (Biswal et al., 1995; Greicius et al., 2003; Fransson, 2005; Fox and Raichle, 2007), MEG (Liu et al., 2010; Brookes et al., 2011; Luckhoo et al., 2012; de Pasquale et al., 2012), and EEG-fMRI techniques (Mantini et al., 2007). The topology of human FC patterns has been studied in detail across different conditions of rest or cognitive tasks (Achard et al., 2006, Bassett et al., 2006). In humans, a consistent set of functional networks (default, dorsal attention, ventral attention, vision, somatomotor, auditory, frontoparietal) have been identified in the resting-state activity across subjects and across multiple sessions using a variety of methods, which can be used to generate whole-brain functional parcellations of the cerebral cortex (Doucet et al., 2011; Power et al., 2011; Yeo et al., 2011; Hacker et al., 2013). Of particular interest is the default network: a set of correlated brain regions that are more activated at rest than during the performance of cognitive goal-directed tasks (Shulman et al., 1997; Raichle et al., 2001; Fox and Raichle, 2007). 
In contrast, single-cell recordings in monkeys indicate that short-range spontaneous correlations between neighboring neurons are low (Ecker et al., 2010), despite a large amount of shared presynaptic inputs. Different mechanisms have been proposed to account for this decorrelation including chaotic dynamics (van Vreeswijk and Sompolinsky, 1996), balance of excitation and inhibition (Renart et al., 2010), and feedback inhibition (Tetzlaff et al., 2012). In theoretical models, these mechanisms all produce the observed low ( $\sim 3 \mathrm{~Hz}$ for excitatory neurons) and irregular (Poisson-like) cortical activity observed in vivo (Burns and Webb, 1976; Softky and Koch, 1993; Wilson et al., 1994).

The aim of this study is to investigate the effect of local decorrelation on both the resting and evoked activity of a large-scale brain model. Resting-state brain models assume that long-range functional correlations emerge from the embedding of local dynamics in the underlying anatomical connectivity structure (Honey et al., 2007, 2009; Ghosh et al., 2008; Deco et al., 2009, 2013a,b; Deco and Hughes, 2012). In general, biophysical resting-state models use single-area dynamical models that, when isolated, emulate spontaneous state activity, i.e., uncorrelated low firing rate activity. However, the long-range anatomical couplings break down the asynchronous state, producing intra-area correlations which are not consistent with the empirical findings. Here, we show that the regulation of the local feedback inhibition provides a mechanism for reconciling the two levels of spontaneous correlation at different spatial scales and we evaluate its functional consequences for both the resting and the evoked global brain dynamics.

\section{Materials and Methods}

Structural connectivity matrix. Neuroanatomical structure was obtained using diffusion spectrum imaging (DSI) data and tractography from five healthy right-handed male human subjects (Hagmann et al., 2008; Honey et al., 2009). The gray matter was subdivided into 998 regions-ofinterest (ROIs) which are grouped into 33 cortical regions per hemisphere (66 areas in total) according to anatomical landmarks (Table 1). White matter tractography was used to estimate the fiber tract density connecting each pair of ROIs, averaged across subjects. Anatomical connectivity among the 66 cortical regions was calculated by summing all incoming fiber strengths to the corresponding ROIs of the target region, and dividing it by its region-dependent number of ROIs, resulting in a nonsymmetric connectivity matrix. This normalization by the number of ROIs, which have approximately the same surface on the cortex, i.e., the same number of neurons, is required because neuronal activity is sensitive to the number of incoming fibers per neuron in the target region. As the dynamical model of one region already takes into account the effect of its internal connectivity (see below), the connection of a region to itself was set to 0 in the connectivity matrix for the simulations.

Empirical functional connectivity. The empirical resting fMRI FC was measured in 48 scanning sessions from 24 right-handed healthy young volunteers ( 15 females, age range $20-31$ years). Subjects were informed about the experimental procedures and provided written informed consent. The study design was approved by the local Ethics Committee of Chieti University. Subjects lay in a supine position and viewed a black screen with a centered red fixation point of 0.3 visual degrees, through a mirror tilted by 45 degrees. Each volunteer underwent two scanning runs of $10 \mathrm{~min}$ in a resting-state condition. Specifically, they were instructed to relax, but to maintain fixation during scanning. The eye position was monitored at $120 \mathrm{~Hz}$ during scanning using an ISCAN eye tracker system. Scanning was performed with a 3T MR scanner (Achieva; Philips Medical Systems) at the Institute for Advanced Biomedical Technologies in Chieti, Italy. Functional images were obtained using T2-weighted echo-planar imaging (EPI) with blood oxygenation level-dependent (BOLD) contrast using SENSE imaging. EPIs (TR/TE $=2000 / 35 \mathrm{~ms}$ ) comprised 32 axial slices acquired continuously in ascending order covering the entire brain (voxel size $=3 \times 3 \times 3.5 \mathrm{~mm}^{3}$ ). For each scanning
Table 1. Names and abbreviations (alphabetically) of the brain regions considered in the human connectome from Hagmann et al. (2008)

\begin{tabular}{ll}
\hline Brain region & Abbreviation \\
\hline Bank of the superior temporal sulcus & BSTS \\
Caudal anterior cingulate cortex & CAC \\
Caudal middle frontal cortex & CMF \\
Cuneus & CUN \\
Entorhinal cortex & ENT \\
Frontal pole & FP \\
Fusiform gyrus & FUS \\
Inferior parietal cortex & IP \\
Isthmus of the cingulate cortex & ISTC \\
Inferior temporal cortex & IT \\
Lingual gyrus & LING \\
Lateral occipital cortex & LOCC \\
Lateral orbitofrontal cortex & LOF \\
Medial orbitofrontal cortex & MOF \\
Middle temporal cortex & MT \\
Paracentral lobule & PARC \\
Parahippocampal cortex & PARH \\
Posterior cingulate cortex & PC \\
Pericalcarine cortex & PCAL \\
Precuneus & PCUN \\
Pars opercularis & POPE \\
Pars orbitalis & PORB \\
Precentralgyrus & PREC \\
Postcentralgyrus & PSTC \\
Pars triangularis & PTRI \\
Rostral anterior cingulate cortex & RAC \\
Rostral middle frontal cortex & RMF \\
Superior frontal cortex & SF \\
Supramarginalgyrus & SMAR \\
Superior parietal cortex & SP \\
Superior temporal cortex & ST \\
Temporal pole & TP \\
Transverse temporal cortex & TT \\
\hline & \\
& \\
\hline
\end{tabular}

run, initial five dummy volumes allowing the MRI signal to reach steadystate were discarded. The next 300 functional volumes were used for the analysis. A three-dimensional high-resolution T1-weighted image (TR/ $\mathrm{TE}=9.6 / 4.6 \mathrm{~ms}$, voxel size $\left.=0.98 \times 0.98 \times 1.2 \mathrm{~mm}^{3}\right)$ covering the entire brain was acquired at the end of the scanning session and used for anatomical reference. Initial data preprocessing was performed using the SPM5 software package (Wellcome Department of Cognitive Neurology, London, UK) running under MATLAB (MathWorks). The preprocessing steps involved the following: (1) correction for slice-timing differences, (2) correction of head-motion across functional images, (3) coregistration of the anatomical image and the mean functional image, and (4) spatial normalization of all images to a standard stereotaxic space (Montreal Neurological Institute; MNI) with a voxel size of $3 \times 3 \times 3$ $\mathrm{mm}^{3}$. Furthermore, the BOLD time series in MNI space were subjected to spatial independent component analysis (ICA) for the identification and removal of artifacts related to blood pulsation, head movement and instrumental spikes and those that correlate with the white matter and CSF patterns (Sui et al., 2009; Mantini et al., 2013). The BOLD artifact removal procedure was performed using the GIFT toolbox (http://mialab.mrn.org/software/gift/index.html). No global signal regression was performed. For each recording session (subject and run), the BOLD time series from the 998 ROIs of the brain atlas (Hagmann et al., 2008) were averaged over the corresponding 66 brain regions. Finally, we concatenated in time the remaining sessions for each parcel and calculated the correlation matrix (FC).

Computational model. In this study we modeled a cortical area as a canonical local network composed of interconnected excitatory and inhibitory neurons coupled through NMDA, AMPA, and GABA synapses. In the large-scale brain model, we assumed that the inter-area connections are constrained by the empirical anatomical matrix that expresses 
the density of white matter fiber tracts connecting two given brain areas. However, because the large-scale spiking model is composed of many thousands of coupled nonlinear differential equations corresponding to each single neuron and synapse, some approximations need to be done to simplify the model. For this, we used a dynamic mean field (DMF) technique (Wong and Wang, 2006) that approximates the average ensemble behavior, instead of considering the detailed interactions between individual neurons. This allow us varying the parameters and, furthermore, to greatly simplify the system of stochastic differential equations by expressing it in terms of the first and second-order statistical moments, means and covariances, of network activity. In the following we present the details of the model and its simplified versions.

Single area spiking model. To show the effect of feedback inhibition on the correlations between intra-area neurons and on the firing rate activity we used a network of integrate-and-fire (IF) spiking neurons with excitatory (AMPA and NMDA) and inhibitory (GABA-A) synaptic receptor types (Deco and Jirsa, 2012). Each local cortical area is modeled by a fully connected recurrent network of a population of $N_{E}$ excitatory pyramidal neurons and a population of $N_{I}$ inhibitory neurons. The dynamical evolution of the IF neuron's membrane potential $V(t)$ is driven by the incoming local excitatory and inhibitory inputs within the same cortical area, long-range excitatory inputs from all other cortical areas, and external inputs. The time evolution of the membrane potential of a given neuron $i$ obeys the following differential equation:

$$
\begin{aligned}
& C_{m} \frac{d V_{i}(t)}{d t}=-g_{m}\left(V_{i}(t)-V_{L}\right) \\
& -g_{A M P A, \text { ext }}\left(V_{i}(t)-V_{E}\right) s^{A M P A, e x t}(t) \\
& -g_{A M P A, r e c}\left(V_{i}(t)-V_{E}\right) \sum_{j=1}^{N_{E}} w_{i j} s_{j}^{A M P A, r e c}(t), \\
& -\frac{g_{N M D A}\left(V_{i}(t)-V_{E}\right)}{1+\lambda_{N M D A} e^{-\beta V_{i}(t)}} \sum_{j=1}^{N_{E}} w_{i j} s_{j}^{N M D A}(t) \\
& -g_{G A B A}\left(V_{i}(t)-V_{I}\right) \sum_{j=1}^{N_{I}} w_{i j} s_{j}^{G A B A}(t)
\end{aligned}
$$

if the membrane potential is below a given threshold $V_{\text {thr }}$. The neuron generates a spike, when the membrane potential reaches the threshold $V_{\text {thr. }}$. The spike is transmitted to other neurons and the membrane potential is instantaneously reset to $V_{\text {reset }}$ and maintained there for a refractory time $\tau_{\text {ref }}$ during which the neuron is unable to produce further spikes. In Equation $1, g_{\mathrm{m}}$ is the membrane leak conductance, $C_{\mathrm{m}}$ is the capacity of the membrane, and $V_{L}$ is the resting potential. The membrane time constant is defined by $\tau_{\mathrm{m}}=C_{\mathrm{m}} / g_{\mathrm{m}}$. The synaptic input current is given by the last four terms on the right hand side of Equation 1. The spikes arriving at the synapse produce a postsynaptic excitatory or inhibitory potential given by a conductance-based model specified by the synaptic receptors, corresponding to: glutamatergic AMPA external excitatory currents, AMPA and NMDA recurrent excitatory currents, and GABAergic recurrent inhibitory currents. The respective synaptic conductances are $g_{\mathrm{AMPA}, \mathrm{ext}}, g_{\mathrm{AMPA}, \mathrm{rec}}, g_{\mathrm{NMDA}, \mathrm{rec}}$, and $g_{\mathrm{GABA}}$, and $V_{E}$ and $V_{I}$ are the excitatory and inhibitory reversal potentials, respectively. The dimensionless parameters $w_{i j}$ of the connections are the synaptic weights defined as following: the recurrent self-excitation within each excitatory population is given by the weight $w_{E E}=w_{+}=1.4$ (for both AMPA and NMDA recurrent synapses); recurrent inhibition and connections from excitatory to inhibitory neurons and vice versa have the weight $w_{I I}, w_{I E}$, and $w_{E I}$, respectively. The gating variables $s_{j}^{i}(t)$ are the fractions of open channels of neurons and are given by the following:

$$
\begin{aligned}
& \frac{d s_{j}^{I}(t)}{d t}=-\frac{s_{j}^{I}(t)}{\tau_{I}}+\sum_{k} \delta\left(t-t_{j}^{k}\right), \text { for } I=\text { AMPA or GABA } \\
& \frac{d s_{j}^{N M D A}(t)}{d t}=-\frac{s_{j}^{N M D A}(t)}{\tau_{N M D A, d e c a y}}+\alpha x_{j}^{N M D A}(t)\left(1-s_{j}^{N M D A}(t)\right)
\end{aligned}
$$

Table 2. Parameters for spiking and the DMF model

\begin{tabular}{lclllr}
\hline \multicolumn{4}{ll}{ Excitatory neurons } & \multicolumn{4}{l}{ Inhibitory neurons } & \multicolumn{2}{l}{ Synapses } \\
\hline$N_{E}$ & $160 \mathrm{neurons}$ & $N_{I}$ & $40 \mathrm{neurons}$ & $V_{E}$ & $0 \mathrm{mV}$ \\
$C_{m}$ & $0.5 \mathrm{nF}$ & $C_{m}$ & $0.2 \mathrm{nF}$ & $V_{1}$ & $-70 \mathrm{mV}$ \\
$g_{m}$ & $25 \mathrm{nS}$ & $g_{m}$ & $20 \mathrm{nS}$ & $\tau_{\text {AMPA }}$ & $2 \mathrm{~ms}$ \\
$V_{L}$ & $-70 \mathrm{mV}$ & $V_{L}$ & $-70 \mathrm{mV}$ & $\tau_{\text {NMDA,rise }}$ & $2 \mathrm{~ms}$ \\
$V_{\text {thr }}$ & $-50 \mathrm{mV}$ & $V_{\text {thr }}$ & $-50 \mathrm{mV}$ & $\tau_{\text {NMDA,decay }}$ & $100 \mathrm{~ms}$ \\
$V_{\text {reset }}$ & $-55 \mathrm{mV}$ & $V_{\text {reset }}$ & $-55 \mathrm{mV}$ & $\tau_{\text {GABA }}$ & $10 \mathrm{~ms}$ \\
$\tau_{\text {ref }}$ & $2 \mathrm{~ms}$ & $\tau_{\text {ref }}$ & $1 \mathrm{~ms}$ & $\alpha$ & $0.5 \mathrm{kHz}$ \\
$g_{\text {AMPA, ext }}$ & $3.37 \mathrm{nS}$ & $g_{\text {AMPA,ext }}$ & $2.59 \mathrm{nS}$ & $\beta$ & 0.062 \\
$g_{\text {AMPA,rec }}$ & $0.065 \mathrm{nS}$ & $g_{\text {AMPA,rec }}$ & $0.051 \mathrm{nS}$ & $\lambda_{\text {NMDA }}$ & 0.28 \\
$g_{\text {NMDA,rec }}$ & $0.20 \mathrm{nS}$ & $g_{\text {NMDA,rec }}$ & $0.16 \mathrm{nS}$ & & \\
$g_{\text {GABA }}$ & $10.94 \mathrm{nS}$ & $g_{\text {GABA }}$ & $8.51 \mathrm{nS}$ & & \\
\hline
\end{tabular}

$$
\frac{d x_{j}^{N M D A}(t)}{d t}=-\frac{x_{j}^{N M D A}(t)}{\tau_{N M D A, \text { rise }}}+\sum_{k} \delta\left(t-t_{j}^{k}\right) .
$$

Where the sums over the index $k$ represent all the spikes emitted by the presynaptic neuron $j$ (at times $t_{j}^{k}$ ); $\tau_{\mathrm{AMPA}}$ and $\tau_{\mathrm{GABA}}$ represent the decay times for AMPA and GABA synapses, and $\tau_{\mathrm{NMDA} \text {,rise }}$ and $\tau_{\mathrm{NMDA} \text {,decay }}$ are the rise and decay times for the NMDA synapses. All neurons in the network receive an AMPA-mediated external background input of uncorrelated Poisson spike trains with a mean rate of $\nu_{0}=2.4 \mathrm{kHz}$. Parameter values of the neurons and the synapses are summarized in Table 2.

The values of the local connections were set to $w_{I I}=w_{E I}=w_{I E}=1$. With these parameter values the spiking activity of the above local network mimics the observed spontaneous activity, i.e., uncorrelated low activity with mean firing rate equal to 2.92 and $7.54 \mathrm{~Hz}$ for the excitatory units and the inhibitory units, respectively. However, when two or more local networks are connected through long-range excitatory connections, the external excitation increases the correlations within the local networks (see below). To compensate for the excess of excitation, we postulate a local regulation mechanism, called feedback inhibition control (FIC), in which the connection weights from inhibitory to excitatory neurons within each cortical area are adjusted to clamp the firing rate at $\sim 3 \mathrm{~Hz}$ for each local excitatory neural population in the large-scale network (see below). In the FIC case, the optimized inhibitory-excitatory weight of cortical area $i$ is noted $w_{E I}=J_{i}$, whereas $w_{I I}=w_{I E}=1$.

Large-scale cortical dynamic mean field model. As we will see below, the adjustment of the local feedback inhibition of each cortical area involves the recursive adaptation of the inhibitory-excitatory weights. This algorithm requires the computation of the global dynamics during a long period of time, until the FIC constraint is satisfied in all areas. Therefore, we used here a reduced DMF (Wong and Wang, 2006) for computing the global dynamics of the whole cortex. The DMF expresses consistently the time evolution of the ensemble activity of the different excitatory and inhibitory neural populations building up the spiking network. In the DMF approach, each population firing rate depends on the input currents into that population. On the other hand, the input currents depend on the firing rates. Hence, the population firing rate can be determined self-consistently by a reduced system of coupled nonlinear differential equations expressing the population firing rates and the respective input currents. The large-scale model interconnects these local subnetworks according the structural connectivity matrix (SC) defined by the neuroanatomical connections between those brain areas in the human, as obtained by DSI and describe above. The inter-area connections are established as long range excitatory synaptic connections either between excitatory pools of different areas only or both between excitatory pools and from excitatory pools to inhibitory the inhibitory pools of different areas (feedforward inhibition). Inter-areal connections are weighted by the strength specified in the neuroanatomical matrix SC, denoting the density of fibers between those regions, and by a global scaling factor $G$. The global scaling factor is a free control parameter that we vary systematically to study the dynamics of the global cortical system.

In brief, the mean field approach considers the diffusion approximation according to which sums of synaptic gating variables (Eq. 1) are replaced by the averaged component and a Gaussian fluctuation term. 
Moreover, the first passage time equation, that gives the mean firing rate of a neuron receiving a noisy input, is approximated by a simple sigmoidal input-output function giving the firing rate as a function of the inputs (Wong and Wang, 2006). Because the synaptic gating variable of NMDA receptors has a much longer decay time constant $(100 \mathrm{~ms})$ than the AMPA receptors, the dynamics of the NMDA gating variable dominates the time evolution of the system, while the AMPA synaptic variable instantaneously reaches its steady-state. We thus have neglected contributions by the AMPA receptors to the local recurrent excitation. All these approximations greatly simplify the model without compromising its performance. Indeed, it has been previously shown that these approximations conserve the first-order (fixed points of the mean activity) and the second-order (correlation structure) statistics of the original largescale spiking model (Deco et al., 2013a) which includes AMPA, NMDA, and GABA synapses. Finally, in the present study long-range AMPAmediated connections are assumed to be instantaneous, thus conduction delays between distant cortical areas are neglected.

The global brain dynamics are described by the following set of coupled nonlinear stochastic differential equations:

$$
I_{i}^{(\mathrm{E})}=W_{E} I_{0}+w_{+} J_{N M D A} S_{i}^{\left({ }^{(E)}\right.}+G J_{N M D A} \sum_{j} C_{i j} S_{j}^{(E)}-J_{i} S_{i}^{(I)}+I_{\text {external }},
$$

$$
\begin{gathered}
I_{i}^{(\mathrm{I})}=W_{I} I_{0}+J_{N M D A} S_{i}^{(E)}-S_{i}^{(I)}+\lambda G J_{N M D A} \sum_{j} C_{i j} S_{j}^{(E)}, \\
r_{i}^{(E)}=H^{(E)}\left(I_{i}^{(E)}\right)=\frac{a_{E} I_{i}^{(E)}-b_{E}}{1-\exp \left(-d_{E}\left(a_{E} I_{i}^{(E)}-b_{E}\right)\right)}, \\
r_{i}^{(\mathrm{I})}=H^{(\mathrm{I})}\left(I_{i}^{(\mathrm{I})}\right)=\frac{a_{I} I_{i}^{(\mathrm{I})}-b_{I}}{1-\exp \left(-d_{I}\left(a_{I} I_{i}^{(\mathrm{I})}-b_{I}\right)\right)}, \\
\frac{d S_{i}^{(E)}(t)}{d t}=-\frac{S_{i}^{(E)}}{\tau_{E}}+\left(1-S_{i}^{(E)}\right) \gamma r_{i}^{(E)}+\sigma v_{i}(t), \\
\frac{d S_{i}^{(\mathrm{I})}(t)}{d t}=-\frac{S_{i}^{(\mathrm{I})}}{\tau_{I}}+r_{i}^{(\mathrm{I})}+\sigma v_{i}(t),
\end{gathered}
$$

where $r_{i}^{(E, I)}$ denotes the population firing rate of the excitatory $(E)$ or inhibitory (I) population in the brain area $i . S_{i}^{(E, I)}$ denotes the average excitatory or inhibitory synaptic gating variable at the local area $i$. The input currents to the excitatory or inhibitory population $i$ is given by $I_{i}^{(E, I)}$. $I_{\text {external }}$ encodes external stimulation for simulating task evoked activity (it is zero for all pools under resting state condition, and 0.02 for those pools excited in a task condition). Furthermore, $w_{+}=1.4$ is the local excitatory recurrence, and $C_{i j}$ is the structural connectivity matrix expressing the neuroanatomical links between the areas $i$ and $j$. The parameter $\lambda$ can be equal to 1 or 0 and indicates whether long-range feedforward inhibition (FFI) is considered $(\lambda=1)$ or not $(\lambda=0)$. Note that in the case of FFI the proportion of excitatory-inhibitory long-range connections and excitatory-excitatory long-range connections is the same. $H^{(E)}$ and $H^{(I)}$ denotes the neuronal input-output functions of excitatory pools and inhibitory pools, respectively. The input-output function converts incoming inputs into firing rates. The kinetic parameters are $\gamma=0.641 / 1000$ (the factor 1000 is for expressing everything in $\mathrm{ms})$, and $\tau_{E}=\tau_{\mathrm{NMDA}}$ and $\tau_{I}=\tau_{\mathrm{GABA}}$. The excitatory synaptic coupling $J_{\mathrm{NMDA}}=0.15(\mathrm{nA})$ and the local feedback inhibitory synaptic coupling $J_{i}$ is 1 for each brain area $i$ in the no-FIC case, and for the FIC case it is adjusted independently, by the algorithm described below. The overall effective external input is $I_{0}=0.382(\mathrm{nA})$ scaled by $W_{E}$ and $W_{I}$, for the excitatory pools and the inhibitory pools, respectively. In Equations 9 and $10 v_{i}$ is uncorrelated standard Gaussian noise and the noise amplitude at each node is $\sigma=0.01(\mathrm{nA})$. Equation 9 is derived from Equations 3 and 4 , by replacing the sum over presynaptic delta-like spikes by the mean firing rate $r_{i}^{(E)}$ and noting that the effective time constant of NMDA is $\tau_{\mathrm{NMDA}}=\alpha \tau_{\mathrm{NMDA}, \text { rise }} \tau_{\mathrm{NMDA}, \text { decay }}=100 \mathrm{~ms}$ (Wong and Wang, 2006). Similarly, Equation 10 is derived from Equation 2. See Table 3 for parameter values. The values of $W_{I}, I_{0}$, and $J_{N M D A}$ were chosen to obtain a low level of spontaneous activity for the isolated local area model. With these
Table 3. DMF model variables

\begin{tabular}{ll}
\hline Excitatory gating variables & Inhibitory gating variables \\
\hline$a_{E}=310\left(\mathrm{nC}{ }^{-1}\right)$ & $a_{l}=615\left(\mathrm{nC}^{-1}\right)$ \\
$b_{E}=125(\mathrm{~Hz})$ & $b_{l}=177(\mathrm{~Hz})$ \\
$d_{E}=0.16(\mathrm{~s})$ & $d_{l}=0.087(\mathrm{~s})$ \\
$\tau_{E}=\tau_{\mathrm{NMDA}}=100(\mathrm{~ms})$ & $\tau_{l}=\tau_{\mathrm{GABA}}=10(\mathrm{~ms})$ \\
$W_{E}=1$ & $W_{l}=0.7$ \\
\hline
\end{tabular}

parameter values the mean spiking activity of the excitatory pool, for an isolate local network $(G=0)$, is equal to $3.0631 \mathrm{~Hz}$. Note that this tuning is necessary because the system of equations (5-10) results from a succession of approximations, among them the neglect of AMPA receptors, which inclusion would otherwise highly complicate the reduced model (Wong and Wang, 2006).

The fixed points of the above dynamical system were calculated by numerically solving the steady-state system of nonlinear equations (Eqs. $5-10$, in the absence of noise) using the MATLAB's fsolve function.

For comparison to the empirical fMRI BOLD functional connectivity matrix, we transformed the simulated excitatory synaptic activity, $S^{(E)}$, to BOLD signals using the Balloon-Windkessel hemodynamic model (Friston et al., 2003; Deco et al., 2013a).

FIC. For an isolated node, with the intrinsic parameters described above, an input to the excitatory pool equal to $I_{i}^{E}-b_{E} / a_{E}=-0.026$; i.e., slightly inhibitory dominated, leads to a firing rate equal to $3.0631 \mathrm{~Hz}$. Hence, in the large-scale model of interconnected brain areas, we aim to constraint in each brain area $(i)$ the local feedback inhibitory weight $J_{i}$ such that $I_{i}^{E}-b_{E} / a_{E}=-0.026$ is fulfilled (with a tolerance of \pm 0.005 , implying an excitatory firing rate between $2.63-3.55 \mathrm{~Hz}$ ). To achieve this, we apply following procedure: we simulate during a period of $10 \mathrm{~s}$ the system of stochastic differential Equations 5-10 and compute the averaged level of the input to the local excitatory pool of each brain area, i.e., $I_{i}^{(E)}$. If $I_{i}^{E}-b_{E} / a_{E}=-0.026$ then we upregulate the corresponding local feedback inhibition $J_{i}=J_{i}+\Delta$; otherwise, we downregulate $J_{i}=J_{i}-\Delta$. We recursively repeat this procedure until the constraint on the input to the local excitatory pool is fulfilled in all 66 brain areas.

Analytical approximation of the activity covariance. To estimate the network's statistics, we approximate deterministic dynamical equations for statistical moments of network's gating variables. This "moments' method" avoids extensive simulations of the entire stochastic system, that otherwise would be required for estimation of the network moments. For this, we express the system of stochastic differential equations $(5-10)$ in terms of the first- and second-order moments of the distribution of synaptic gating variables: $\mu_{i}^{(m)}$, the expected mean gating variable of a given local neural population of type $m$ (where $m=E$ or $I$ ) of the cortical area $i$, and $P_{i j}^{(m n)}$, the covariance between gating variables of neural populations of type $m$ and $n$ of local cortical areas $i$ and $j$, respectively. The moments are defined as follows:

$$
\begin{gathered}
\mu_{i}^{(m)}(t)=\left\langle S_{i}^{(m)}(t)\right\rangle \\
P_{i j}^{(m n)}(t)=\left\langle\left[S_{i}^{(m)}(t)-\mu_{i}^{(m)}(t)\right]\left[S_{j}^{(n)}(t)-\mu_{j}^{(n)}(t)\right]\right\rangle .
\end{gathered}
$$

Where the angular brackets $<$. $>$ denote the average over realizations. In vector form, the system of equations writes as follows:

$$
\frac{d}{d t}\left(\begin{array}{c}
\vec{S}^{(E)} \\
\vec{S}^{(I)}
\end{array}\right)=\left(\begin{array}{l}
f^{(E)}\left(\vec{S}^{(E)}, \vec{S}^{(I)}\right) \\
f^{(I)}\left(\vec{S}^{(E)}, \vec{S}^{(I)}\right)
\end{array}\right)
$$

where $\vec{S}=\left\{\vec{S}^{(E)}, \vec{S}^{(I)}\right\}=\left\{S_{1}^{(E)}, \ldots, S_{N}^{(E)}, S_{1}^{(I)}, \ldots, S_{N}^{(I)}\right\}, f_{k}^{(E)}\left(\vec{S}^{(E)}, \vec{S}^{(I)}\right)=-\frac{S_{k}^{(E)}}{\tau_{E}}+$ $\left(1-S_{k}^{(E)}\right) \gamma H^{(E)}\left(I_{k}^{(E)}\right)$ and $f_{k}^{(I)}\left(\vec{S}^{(E)}, \vec{S}^{(I)}\right)=-\frac{S_{k}^{(I)}}{\tau_{I}}+H^{(I)}\left(I_{k}^{(I)}\right)$ for $k=1, . ., N$.

Taylor expanding $\vec{S}$ around $\vec{\mu}=\langle\vec{S}\rangle$; i.e., $S_{i}^{(m)}=\mu_{i}^{(m)}+\delta S_{i}^{(m)}$, up to the first order, we get the following: 


$$
f_{k}^{(m)}(\vec{S})=f_{k}^{(m)}(\vec{\mu})+\sum_{i} \frac{\partial f_{k}^{(m)}}{\partial S_{i}^{(E)}}(\vec{\mu}) \cdot \delta S_{i}^{(E)}+\sum_{i} \frac{\partial f_{k}^{(m)}}{\partial S_{i}^{(I)}}(\vec{\mu}) \cdot \delta S_{i}^{(I)} .
$$

Using this approximation, tacking the average over realizations, and noting that:

$$
\left\langle\delta S_{i}^{(m)}(t)\right\rangle=\langle v(t)\rangle=0,
$$

we obtain the motion equations for the means of the gating variables and the covariance of the fluctuations around the mean. For the mean values:

$$
\begin{gathered}
\frac{d \mu_{i}^{(E)}}{d t}=\frac{d}{d t}\left\langle\vec{S}^{(E)}\right\rangle=-\frac{\mu_{i}^{(E)}}{\tau_{E}}+\left(1-\mu_{i}^{(E)}\right) \gamma H^{(E)}\left(u_{i}^{(E)}\right) \\
\frac{d \mu_{i}^{(I)}}{d t}=\frac{d}{d t}\left\langle\vec{S}^{(I)}\right\rangle=-\frac{\mu_{i}^{(I)}}{\tau_{I}}+H^{(I)}\left(u_{i}^{(I)}\right) .
\end{gathered}
$$

Where $u_{i}^{(m)}$ is the mean input current to the neural population $m=E, I$ of cortical area $i$, defined as follows:

$$
u_{i}^{(E)}=W_{E} I_{0}+w_{+} J_{N M D A} \mu_{i}^{(E)}+G J_{N M D A} \sum_{j} C_{i j} \mu_{j}^{(E)}-J_{i} \mu_{i}^{(I)}+I_{\text {external }}
$$

$$
u_{i}^{(I)}=W_{I} I_{0}+J_{N M D A} \mu_{i}^{(E)}-\mu_{i}^{(I)}+\lambda G J_{N M D A} \sum_{j} C_{i j} \mu_{j}^{(E)} .
$$

For the fluctuations:

$$
\begin{aligned}
\frac{d}{d t}\left\langle\delta S_{i}^{(m)} \delta S_{j}^{(n)}\right\rangle= & \sum_{p=E, I} \sum_{k} \frac{\partial f_{i}^{(m)}(\vec{\mu})}{\partial S_{k}^{(p)}}\left\langle\delta S_{k}^{(p)} \delta S_{j}^{(n)}\right\rangle \\
& +\sum_{p=E, I} \sum_{k} \frac{\partial f_{i}^{(m)}(\vec{\mu})}{\partial S_{k}^{(p)}}\left\langle\delta S_{k}^{(p)} \delta S_{i}^{(m)}\right\rangle+\sigma^{2}\left\langle\boldsymbol{v}_{i} \boldsymbol{v}_{j}\right\rangle .
\end{aligned}
$$

Let $\mathbf{P}$ being the covariance matrix between gating variables. $P$ is a block matrix defined as follows:

$$
\mathbf{P}=\left\langle\vec{S} \vec{S}^{T}\right\rangle=\left[\begin{array}{ll}
P^{(E E)} & P^{(E I)} \\
P^{(I E)} & P^{(I I)}
\end{array}\right] \text {, where the superscript } T \text { is the transpose. }
$$

Using Equation 20, we obtain the motion equation of the covariance matrix:

$$
\frac{d \mathbf{P}}{d t}=\mathbf{A P}+\mathbf{P A}^{\mathrm{T}}+\mathbf{Q}_{\mathbf{n}}
$$

Where $\mathbf{Q}_{\mathbf{n}}$ is the covariance matrix of the noise (which is diagonal for uncorrelated white noise) and A is the Jacobian matrix of the system. A is a block matrix defined as follows:

$$
\mathbf{A}=\left[\begin{array}{ll}
A^{(E E)} & A^{(E I)} \\
A^{(I E)} & A^{(I I)}
\end{array}\right], \text { where } A_{i j}^{(m n)}=\left\{\frac{\partial f_{i}^{(m)}(\vec{\mu})}{\partial S_{j}^{(n)}}\right\} .
$$

Finally, note that the above derivatives can be written as follows:

$$
\begin{aligned}
\frac{\partial f_{i}^{(m)}(\vec{\mu})}{\partial S_{j}^{(n)}}=[ & \left.-\frac{1}{\tau_{m}}-\gamma H^{(E)}\left(u_{i}^{(E)}\right) \delta_{E m} \delta_{E n}\right] \delta_{i j} \\
& +\left[1+\left(1-\mu_{i}^{(E)}\right) \gamma \delta_{E m} \delta_{E n}\right] \frac{\partial H^{(m)}\left(u_{i}^{(n)}\right)}{\partial u} \cdot K_{i j}^{(m n)} .
\end{aligned}
$$

Where $\mathbf{K}=\left[\begin{array}{ll}K^{(E E)} & K^{(E I)} \\ K^{(I E)} & K^{(I I)}\end{array}\right]$, with $K_{i j}^{(E I)}=-J_{i} \delta_{i j}, K^{(I E)}=J_{N M D A} \mathbf{I}+$ $\lambda G J_{N M D A} \mathbf{C}, K^{(I I)}=-\mathbf{I}$, and $K^{(E E)}=w_{+} J_{N M D A} \mathbf{I}+G J_{N M D A} \mathbf{C}$, where $\mathbf{I}$ and $\mathrm{C}$ are the $\mathrm{N}$-by- $\mathrm{N}$ identity matrix and anatomical connectivity matrix, respectively.

Equations 20-22 indicate that the covariance of gating variables is determined by both the underlying connectivity and the dynamics. In the stationary regime, the covariance matrix of fluctuations around the spontaneous state is given by the algebraic equation:

$$
\mathbf{A P}+\mathbf{P A}^{\mathrm{T}}+\mathbf{Q}_{\mathbf{n}}=0,
$$

which can be solved using the Eigen-decomposition of the Jacobian matrix evaluated at the fixed points: $\mathbf{A}=\mathbf{L D L} \mathbf{L}^{-1}$, where $\mathbf{D}$ is a diagonal matrix containing the eigenvalues of $\mathbf{A}$, denoted $\lambda_{i}$, and the columns of matrix $\mathbf{L}$ are the eigenvectors of $\mathbf{A}$. Multiplying Equation 23 by $\mathbf{L}^{-1}$ from the left and by $\mathbf{L}^{-\dagger}$ from the right (the superscript dagger being the conjugate transpose) we get the following:

$$
\mathbf{P}=\mathrm{LML}^{\dagger},
$$

Where $M$ is given by the following: $M_{i j}=-\tilde{Q}_{i j} /\left(\lambda_{i}+\lambda_{j}^{*}\right)$, and $\tilde{\mathbf{Q}}=\mathrm{L}^{-1} \mathbf{Q}_{\mathbf{n}} \mathbf{L}^{-\dagger}$.

Thus, the covariance matrix of spontaneous fluctuations is determined by the eigenvalues of the Jacobian matrix, which, in turn, is related to the connectivity matrix and the dynamics. Hence, Equation 24 provides a direct link between the correlation structure, the underlying connectivity, and dynamics. Indeed, the interpretation of Equation 24 is that the input covariance $(\mathbf{M})$ is propagated through the dynamical system and is mapped to its approximated output $(\mathbf{P})$.

Finally, to compare the results from the above moments' method to the empirical functional connectivity, we estimated the correlation matrix, noted $\mathbf{Q}$, between the gating variables of the excitatory populations, defined as follows: $Q_{i j}=P_{i j}^{(E E)} / \sqrt{P_{i i}^{(E E)} \times P_{j j}^{(E E)}}$.

Power spectrum of linear fluctuations. The power spectrum of fluctuations around the fixed points, $\Phi(\omega)$, can be obtained by, first, writing the equation of the fluctuations around the fixed points, given by the following:

$$
\frac{d \delta \vec{S}(t)}{d t}=A \delta \vec{S}(t)+\sigma \vec{v}(t)
$$

Taking the Fourier transform of Equation 25 we get the following:

$$
-i \omega \delta \tilde{S}(\omega)=A \delta \tilde{S}(\omega)+\sigma \tilde{v}(\omega) .
$$

Hence, $\delta \tilde{S}(\omega)=-(J+i \omega)^{-1} \sigma \tilde{v}(\omega)$. The power spectrum being the expectation of the Fourier transformed autocorrelation function, we get the following:

$$
\Phi(\omega)=\left\langle\delta \tilde{S}(\omega) \delta \tilde{S}(\omega)^{\dagger}\right\rangle=\sigma^{2}(A+i \omega)^{-1}\left(A^{T}-i \omega\right)^{-1} .
$$

Thus, the power spectrum of $\delta S_{k}$ is as follows:

$$
\Phi_{k}(\omega)=\sigma^{2} \sum_{l}\left|\left(J_{k l}+i \omega \delta_{k l}\right)^{-1}\right|^{2} .
$$

Model prediction of the empirical connectivity. As a measure of similarity between two FC matrices we used the uncentered Pearson correlation coefficient between corresponding elements of the upper triangular part of the matrices, defined as follows:

$$
r_{c}=\frac{\langle X Y\rangle}{S D(X) S D(Y)} .
$$

Where $X$ and $Y$ are the vectors containing the upper triangular values of each matrix, respectively, and SD is the standard deviation. For FC matrices, because the sampling distribution of correlation coefficients is not normally distributed, we used the Fisher's $z$-transformation to convert the matrix elements before applying any subsequent test. The uncentered correlation takes into account the difference in the mean values of the FC matrices. Confidence intervals were obtained using bootstrap resampling (1000 resamples).

For comparing a structural connectivity matrix and a FC matrix we used the centered Pearson correlation defined as follows:

$$
r_{c}=\frac{\langle(X-\langle X\rangle)(Y-\langle Y\rangle)\rangle}{S D(X) S D(Y)} .
$$

Entropy of binary evoked patterns. The response of the large-scale network to different stimuli was analyzed to investigate its behavior under 
hypothetical task conditions and its information capacity. Stimuli were constructed by imposing an external input $I_{\text {external }}=0.02$ (1) to the excitatory population of $10 \%$ of the brain areas, randomly selected, or (2) to both the excitatory and inhibitory populations of $10 \%$ of the brain areas, randomly selected. The procedure was repeated 1000 times and the resulting steady-state evoked response of the network were stored. Evoked patterns were binarized by imposing a threshold above which the excitatory firing rate of a given area was set to 1 and, otherwise, it was set to 0 (only the firing rate of the excitatory pools were used, thus the binary evoked pattern is 66-dimensional). The threshold was defined as $\theta . \mathrm{SD}$, where SD is the standard deviation across all evoked patterns and the multiplier $\theta$ is a scalar parameter. In this way we obtained binary patterns containing 66 entries. The entropy of the set of evoked binary patterns $R$ is defined as follows:

$$
H(R)=-\sum_{i=1}^{n} p_{i} \log _{2} p_{i}
$$

Where $n$ is the number of unique patterns and $p_{i}$ is the probability that pattern $i$ is observed. Only patterns with at least one non-null entry were analyzed.

To remove the sampling bias, we corrected the entropy values by using a quadratic extrapolation procedure (Treves and Panzeri, 1995; Shew et al., 2011). This procedure evaluates the entropy for random samples of fractions $f$ from the full set of $K$ patterns to estimate the sample entropy $H(f)$. It has been shown that the bias of the entropy can be accurately approximated as second order expansions in $1 /(f K)$; i.e., $H(f)=H_{0}+$ $k_{1} \cdot(f K)^{-1}+k_{2} \cdot(f K)^{-2}$, where $H_{0}$ is the true value of the entropy and $k_{1} \cdot(f K)^{-1}+k_{2} \cdot(f K)^{-2}$ is the bias (Treves and Panzeri, 1995). Fitting (least-square-error procedure) the sample entropy $H(f)$ with a quadratic function of $1 /(f K)$, provides the estimate of the unbiased entropy $H_{0}$. Additionally, the fitting provides confidence intervals.

Fisher information. We quantified the encoding accuracy of the largescale network response by calculating the Fisher information (FI). Assuming that the network response is well described by a multivariate Gaussian distribution, i.e., for weak noise, FI can be written as the sum of two terms: $\mathrm{FI}=\mathrm{FI}_{\text {mean }}+\mathrm{FI}_{\text {cov }}$ (Abbott and Dayan, 1999), where

$$
\begin{gathered}
F I_{\text {mean }}(\Delta I)=\overrightarrow{\boldsymbol{\mu}}^{\prime}(\Delta I)^{\mathrm{T}} \mathbf{P}(\Delta I)^{-1} \overrightarrow{\boldsymbol{\mu}}^{\prime}(\Delta I) \\
F I_{\text {cov }}(\Delta I)=\frac{1}{2} \operatorname{Trace}\left(\left[\mathbf{P}^{\prime}(\Delta I) \mathbf{P}(\Delta I)^{-1}\right]^{2}\right) .
\end{gathered}
$$

Where $\overrightarrow{\boldsymbol{\mu}}(\Delta I)$ and $\mathbf{P}(\Delta I)$ are the stationary mean synaptic activity and the covariance matrix evoked by an excitatory stimulus of intensity $\Delta I$, respectively; $\overrightarrow{\boldsymbol{\mu}}^{\prime}(\Delta I)$ and $\mathbf{P}^{\prime}(\Delta I)$ are the first derivatives with respect to the stimulus, evaluated at $\Delta I$. The values of $\overrightarrow{\boldsymbol{\mu}}(\Delta I)$ and $\mathbf{P}(\Delta I)$ were estimated using the moment's equations (Eqs. 16-19, for the mean, and Eq. 24 for the covariance), in the presence of an applied stimulus, i.e., $I_{\text {external }}=\Delta I$. Only the excitatory parts of $\overrightarrow{\boldsymbol{\mu}}$ and $\mathbf{P}$ were considered for calculating the FI. In this study, the stimulus was applied to the excitatory population of both right and left lateral occipital cortex (LOCC), i.e., $I_{\text {external }}=\Delta I$ for rLOCC and ILOCC, and $I_{\text {external }}=0$ for all other cortical areas. The intensity of the stimulus varied from $\Delta I=0.02$ to $\Delta I=0.1$ in steps of 0.001 .

Betweenness centrality. The centrality of a node within the anatomical network was quantified using the betweenness centrality measure. This network measures expresses the number of shortest paths that pass through a given node. For calculating the betweenness centrality we binarized the anatomical matrix $\mathbf{C}$ by imposing a threshold equal to 0.05 above which the anatomical coupling was set to 1 and, otherwise, it was set to 0 .

\section{Results}

\section{Local feedback inhibition control}

In the present study we investigated the effect of regulating the local feedback inhibition on the long-range resting correlations between cortical areas. For this, we used a large-scale model, composed of local networks, or "nodes," representing cortical areas, interconnected by the anatomical connectivity matrix, or

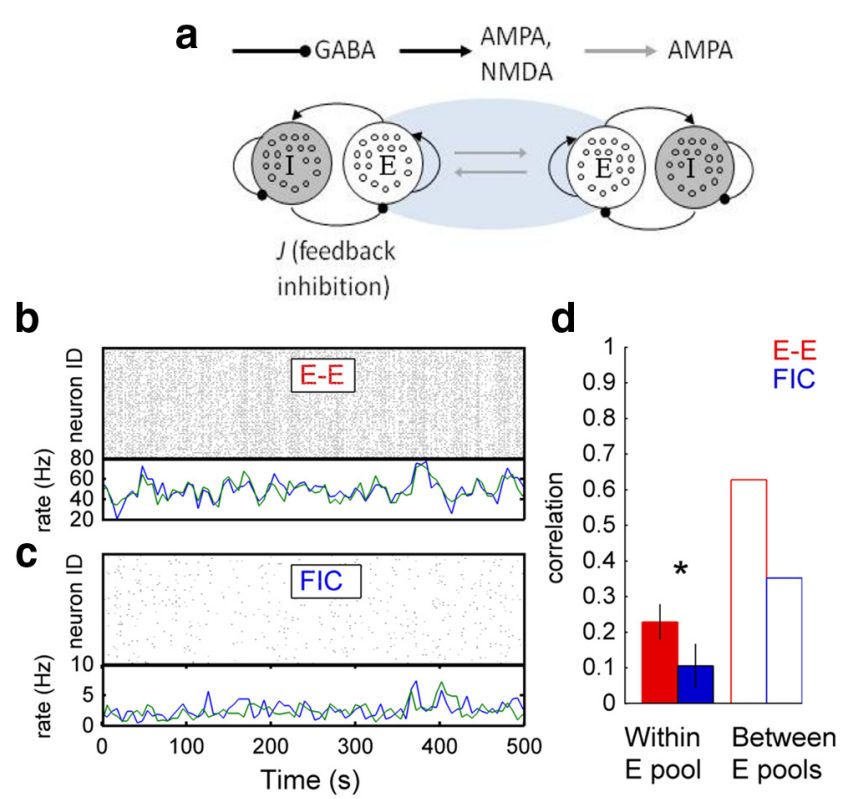

Figure 1. The effect of FIC on the spontaneous spiking activity of two coupled model areas $\boldsymbol{a}$, Two network brain areas model with symmetric connections through their corresponding excitatory pools. The local brain area models consist of $80 \%$ excitatory neurons and $20 \%$ inhibitory neurons recurrently connected such that when isolated the network emulates the neurophysiological characteristics of the empirical observed spontaneous state, i.e., low correlations between the spiking activities of the neurons and low firing rate at $3 \mathrm{~Hz}$. In the FIC scenario, the feedback inhibition is adjusted to compensate the extra excitation the each excitatory pool receive when the brain areas model are connected. $\boldsymbol{b}, \boldsymbol{c}$, Spiking activity of excitatory units from one brain area without FIC ( $\boldsymbol{b}$, top) and with FIC ( $\boldsymbol{c}$, top). Due to symmetry, the spiking activity in the other brain area has the same statistics. The averaged activities of both excitatory pools are plotted in blue and green, for the model without FIC $(\boldsymbol{b}$, bottom) and the model with FIC ( $\boldsymbol{c}$, bottom). $\boldsymbol{d}$, Filled bars, Mean correlation coefficient ( \pm SEM) across all pairwise correlations within one excitatory pool, without and with FIC (red and blue, respectively). Open bars, Correlation between the mean firing rates of both excitatory pools, without and with FIC (red and blue, respectively).

"structural connectivity," between those cortical areas (Deco and Jirsa, 2012; Deco et al., 2013a). Large-scale anatomical connectivity data were obtained using DSI and tractography techniques (Hagmann et al., 2008; see Materials and Methods). Isolated local brain area models consist of $80 \%$ IF excitatory neurons and $20 \%$ inhibitory IF neurons recurrently connected (all-to-all). Intraarea connection weights were such that when isolated the network emulates the neurophysiological characteristics of the empirical observed spontaneous state, i.e., low correlations between the spiking activity of the neurons and low firing rate at $\sim 3$ $\mathrm{Hz}$ (see Materials and Methods for details; Deco and Jirsa, 2012). When the different brain areas are coupled, however, the additional excitatory input injected into each brain area by the longrange pathways breaks the delicate local balance of excitation and inhibition and induces both local correlations and elevated local firing rate. To show this effect, we considered an example case composed of two interconnected single brain areas (Fig. 1a). Connections between the two brain areas were symmetric. The introduction of coupling between nodes leads to high synchronized activity in the excitatory pool (Fig. $1 b$, top). Our working hypothesis is that local feedback inhibition compensates the excess of excitation and decorrelates the activity. To test this, we used a learning algorithm to optimize the strength of the local inhibitory feedback for each brain area (see Materials and Methods) such that all excitatory pools have a low firing rate $\sim 3 \mathrm{~Hz}$. This optimization, or "feedback inhibition control," effectively reduces the intra-area correlations (the mean correlation coeffi- 
cient is $0.11 \pm 0.06$ and $0.23 \pm 0.05$ with and without FIC, respectively, $p<10^{-10}$, $t$ test), but, interestingly, allows for a moderate correlation between the mean firing rates of both excitatory pools (equal to 0.35 and 0.63 with and without FIC, respectively; Fig. $1 c, d$ ). In other words, under FIC, "microscopic" activity is partially decorrelated while "mesoscopic" (population level) quantities covary.

This is the key manipulation that we study in the large-scale model. Before proceeding any further, it is important to note that, in the previous example, although the firing rate is maintained constant, manipulation of the inhibitory feedback changes the eigenvalues of the local network, and, thus, modifies the dynamics.

\section{Large-scale models with and without FIC: stationary states}

In the large-scale cortical network, consisting on coupled cortical areas, we compensated the local feedback inhibition weights $\left(J_{i}\right)$, as previously, to clamp the firing rate $\sim 3 \mathrm{~Hz}$ for each local excitatory neural population $i$ (see Materials and Methods). Because this local compensation mechanism, achieved through recursive adjustments of $J_{i}$, is computationally expensive, we used a DMF to reduce the large-scale spiking network (Wong and Wang, 2006; see Materials and Methods). The DMF ignores the interaction between single neurons within a cortical area and instead considers the ensemble mesoscopic dynamics. By reducing the number of variables, the DMF model allows for the optimization of feedback inhibition weights (Fig. 2a,b).

In the following we compared the fixed points of the model with long-range excitatory-excitatory connections (E-E model) and the model with long-range excitatory-excitatory connections and local feedback inhibition regulation (FIC model). We also consider a third model in which the firing rate within each cortical region is maintained low through long-range connections from the excitatory populations to the inhibitory populations (feedforward inhibition,) in the same proportion as long-range excitatory-excitatory connections and according to the anatomical connectivity (see Materials and Methods).

In all models the inter-area coupling is scaled by a single global parameter, $G$, that changes the network from weakly to strongly connected and determines the dynamical state of the system. We first calculated the bifurcation diagrams characterizing the stationary states of the brain system for the three models (Fig. $2 c, d$ ) as a function of $G$. In all cases we plot, for the different possible states, the maximal firing rate activity across all excitatory populations. The E-E model presents a bifurcation at $G=1.47$ separating two qualitatively different topologies of the attractor landscape. For small values of the global coupling $G$, only one stable state exists, characterized by a firing activity that increases as $G$ increases (stable branch). For $G$ larger than the critical value b
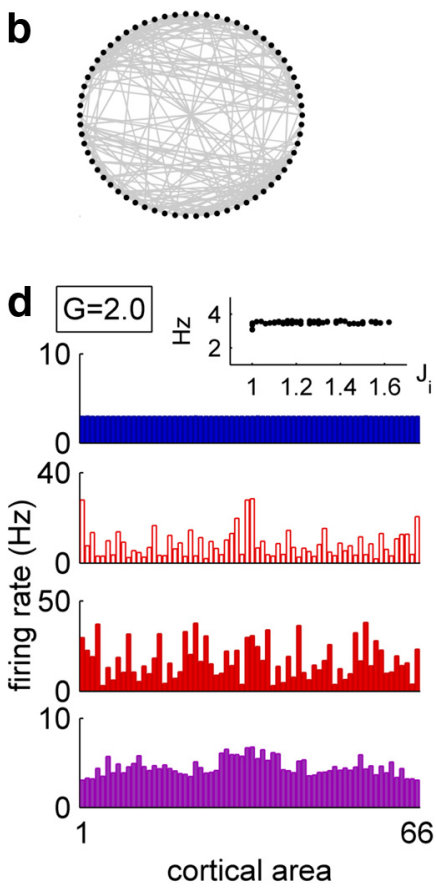

Figure 2. The effect of FIC on the spontaneous mean-field activity of the large-scale brain model. $\boldsymbol{a}$, A dynamic mean field (DMF) connections between the excitatory pools in those areas. In the case of the model with feedforward inhibition (FFI) long-range synaptic AMPA-mediated instantaneous connections from the excitatory pool of a given area to the inhibitory pool of a different area were also considered (dashed arrows). Inter-areal connections are weighted by the strength specified in the pool of each local brain area has a low firing rate $\sim 3 \mathrm{~Hz}$. $\boldsymbol{b}$, Adjacency matrix of the neuroanatomical connectivity. $\boldsymbol{c}$, Attracto (E-E.long-range excitatory-excitatory 列 作 a function of $G$. $\boldsymbol{d}$, The stationary-state of excitatory pools (firing rate vector of dimension $N=66$ ) is shown for $G=2.0$ for the three large-scale DMF models. Blue, FIC model (stable state); the inset shows the stationary firing rates as a function of the local feedback inhibition strength; red, E-E model (stable state); red, open bars, E-E model (unstable state); magenta, FFI model (stable-state).

1.47 a bifurcation occurs and a second fixed point appears which is not stable (unstable branch). The FIC model has only one stable state of low firing activity in all cortical areas, for $G<$ 4.45. For larger values of $G$, long-range interactions are too strong to be compensated by FIC and the activity diverges. In the FFI model the maximum firing rate monotonically increases as a function of $G$.

\section{Feedback inhibition control improves the model's FC prediction}

We next investigate the structure of the emergent correlations from the three global brain models. The predictions of the models were evaluated using the empirical human resting-state FC, obtained by averaging the results across $48 \mathrm{fMRI}$ scanning sessions from 24 healthy human subjects and projected to the same parcellation adopted for the anatomical structural matrix (SC). For comparison with the empirical data, we considered the FC of simulated BOLD signals which are obtained by transforming the model synaptic activity through a hemodynamic model (see Materials and Methods). We calculated the similarity between the 
a

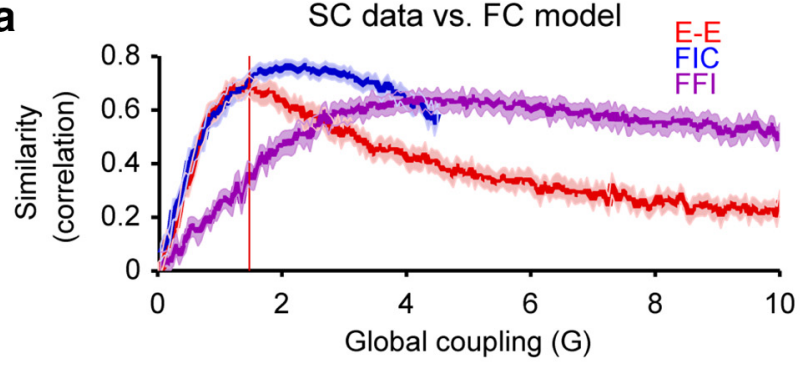

b
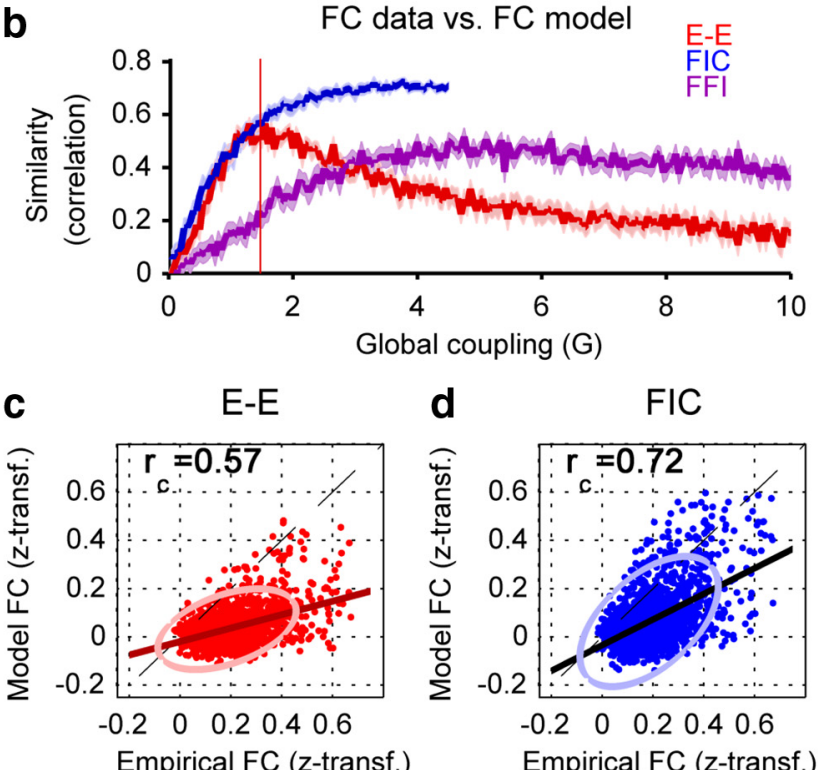

d $\quad \mathrm{FIC}$

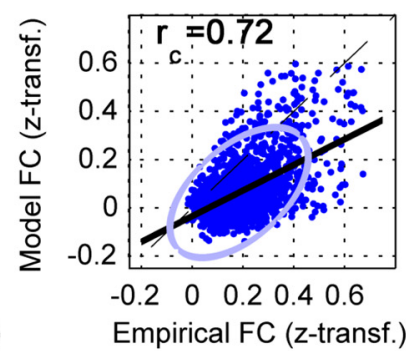

e

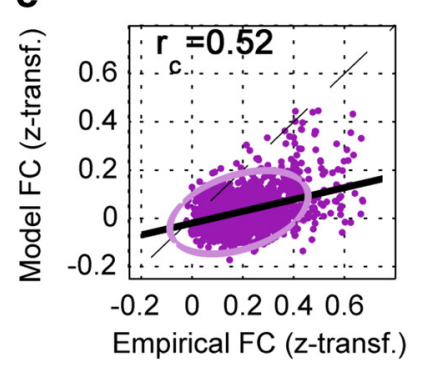

f

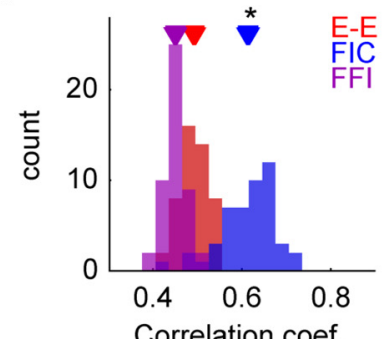

Figure 3. Model prediction of the empirical functional connectivity. $\boldsymbol{a}$, The similarity (correlation coefficient) between the empirical anatomical connectivity (SC) matrix and the model FC was calculated, as a function of the global coupling parameter $G$, for the three different models. In the E-E model, the highest correlation is achieved at the edge of the bifurcation (dashed vertical red line). Shaded areas represent the $95 \%$ confidence interval of the similarity measure. $\boldsymbol{b}$, Similarity (correlation coefficient) between the empirical fMRI FC and the model FC as a function of the global coupling parameter $G$, for the three models. Shaded areas represent the $95 \%$ confidence interval of the similarity measure. $c-\boldsymbol{e}$, Comparison between the empirical FC matrix and the $\mathrm{FC}$ generated by each of the three models, $\mathrm{E}-\mathrm{E}(\boldsymbol{c}), \mathrm{FIC}(\boldsymbol{d})$, and FFI $(\boldsymbol{e})$, for the corresponding optimal values of $G$, equal to $G=1.3, G=3.3$, and $G=4.7$, respectively. Solid lines indicate linear regressions. Ellipses are $95 \%$ confidence ellipses. $r_{c}$ indicates the level of similarity reach for each model. $f$, For each individual fMRI scanning session the FC was predicted after optimization of the parameter $G$ using the $F($ matrix averaged over the remaining 47 scanning sessions. The distribution of similarity measures is presented for each of the three models. The mean similarity is significantly higher for the FIC model compared with the mean similarity of models $\mathrm{E}-\mathrm{E}$ and FFI (ANOVA, ${ }^{*} p<10^{-7}$ ).

model FC and SC, and the similarity between the model FC and the empirical FC, for each model (Fig. $3 a, b$; see Materials and Methods). Importantly, note that, for a given $G$, the large-scale underlying connectivity (i.e., $G \times \mathrm{SC}$ ) is the same for the three models, while the local connectivity is different for the FIC model. We found that, in the E-E, the underlying structural connectivity is maximally expressed in the simulated FC at the edge of the critical value of $G$ (Fig. $3 a$, red trace). Similarly; the empirical FC is optimally fitted by the model near criticality (Fig. $3 b$, red trace). For the FFI model and for the network with FIC, the region where the SC is maximally expressed by the simulated FC and where the empirical data are optimally fitted is much broader (Figs. 3a,b, blue and magenta traces). Furthermore, the level of agreement between the model and the data reached at the maximum is improved in the FIC case.

We further compared the FC of the two models for the corresponding optimal value of $G$, equal to $1.3,4.7$, and 3.3 for the $\mathrm{E}-\mathrm{E}$ model, the FFI model, and the FIC model, respectively (Fig. $3 c-$ e). The scatter plot between the model and the empirical correlation coefficients is closer to the identity line for the FIC model than for the two other models, as can be appreciated by linear regression of the data. The agreement between the model FC and the empirical FC is significantly higher for the FIC model than both for the E-E model ( $p<10^{-7}$ Meng's $z$ test for dependent correlations (Mz-test) and for the FFI model $\left(p<10^{-7}, \mathrm{M} z\right.$ test). We also tested the ability of each model to predict the FC of individual fMRI scanning sessions. For this we used the following jackknife procedure: for each of the $n=48$ scanning sessions we calculated the FC and we computed the agreement between the single-session FC and the FC of each model for which $G$ was optimized using the averaged FC over the remaining $n-1$ sessions. The procedure was repeated such that each scanning session was held out once. The resulting distributions of similarity values for each model are shown in Figure $3 f$. The FIC model gives significantly better predictions of the single-session FC matrices than both the E-E model and the FFI model (ANOVA, $p$ $<10^{-7}$ ).

Moreover, we further quantified the agreement between the simulated FC matrices and the empirical FC by comparing their first principal component (PC), or "dominant spatial mode". For a given covariance matrix, the dominant spatial mode is given by the first eigenvector of the matrix. We calculated the vector projection between the first PC of the empirical data and the first PC of the three models, calculated for the corresponding optimal values of $G$, as previously (Fig. 4). We found that, under FIC, the network approximates better the first PC of the empirical data than do the E-E model and the FFI model.

Altogether, the above results show that the quality of the optimal fitting is better under the FIC condition compared with the unconstrained case. One could argue that the increase in the FC prediction for the FIC model is merely due to the optimization of the connection weights, which makes the model more complex than the other two models. However, note that the parameters of the FIC model where optimized to bound the firing rate of each network node only and not in the optimization of the prediction of the empirical FC which uses only one free parameter $(G)$ as for the other two models.

\section{Correlation of linear noise fluctuations, an analytical approximation}

The reduction of the spiking network through dynamic mean field approximation allowed investigating the large-scale model under the FIC condition. However, the nonlinear and stochastic nature of the DMF equations hinders analytical insights and examination of the dynamics needs to be treated numerically. In particular, estimation of second-order statistic of large networks requires long and multiple time-consuming numerical simulations. In the following, we further reduce the dynamical system 


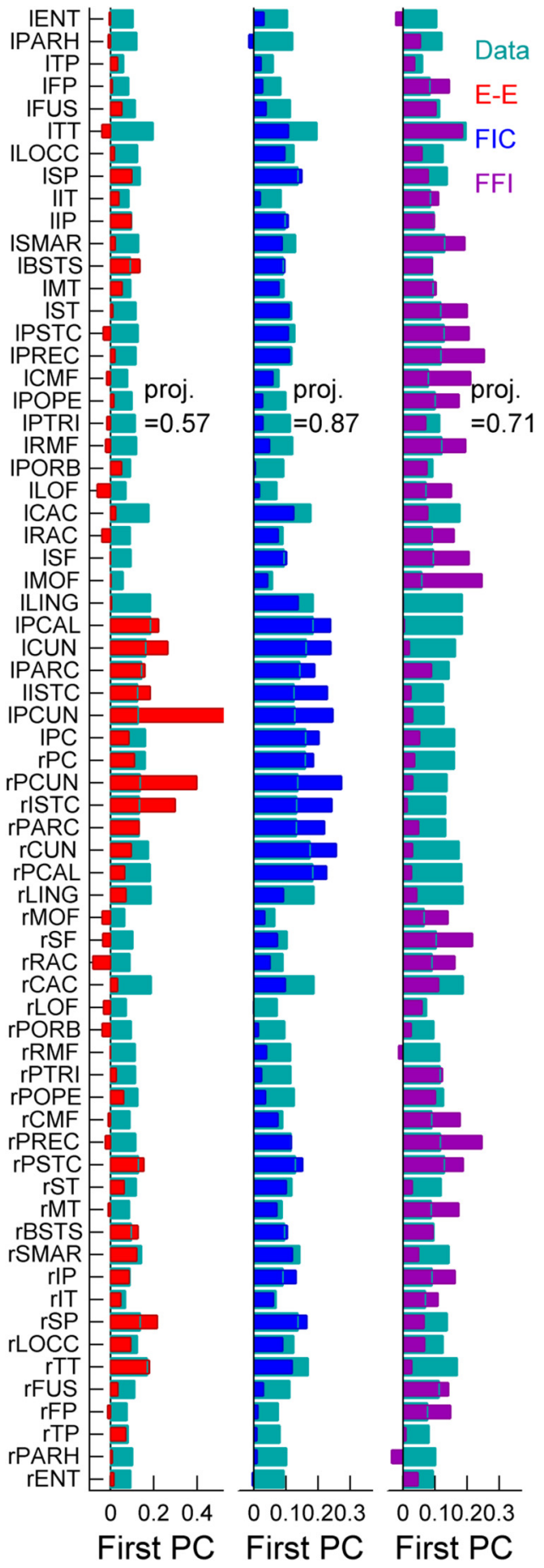

Figure 4. Prediction of the principal mode. The first $\mathrm{PC}$ of the empirical covariance matrix of BOLD signals (turquoise) and the first $P C$ of the model covariance matrix generated by each of the three models, for the corresponding optimal values of $G$. The projection (scalar product) between the first $\mathrm{PC}$ of the empirical data and the first $\mathrm{PC}$ of model data are equal to $0.57 \pm$ $0.05,0.87 \pm 0.02$, and $0.71 \pm 0.03$ for the $E-E$, FIC, and FFI models, respectively.

by expressing it in terms of the first and second-order statistics, means and covariances, of the distribution of gating variables and by deriving deterministic differential equations for these statistics (see Materials and Methods). Briefly, the moments' equations for the reduced nonlinear DMF are derived by Taylor expanding the dynamical equations around the mean values of gating variables and expressing motion equations for the covariances of the fluctuations around the means. This analytical simplification is es- sential, because it provides an explicit link between structure, dynamics, and FC. Indeed, within this linear approximation, the covariance matrix of spontaneous fluctuations is determined by the eigenvalues of the Jacobian matrix (A) of the large-scale network, which in turn is related to the local and large-scale connectivity matrix and the dynamics (see Materials and Methods). Hence, because manipulating the feedback inhibition strength within local networks changes the eigenvalues of $\mathbf{A}$, the correlation structure is modified, even though the firing rates of all model cortical areas remain constant. Using this linear approximation, we calculated the similarity between the correlation matrix of excitatory gating variables, noted $\mathbf{Q}$, and the empirical FC matrix. Note that, in this case, the model correlations matrix is not based on BOLD signals, but on the excitatory gating variable of all cortical areas. We found that the agreement between $\mathbf{Q}$ and the empirical FC is higher for the FIC model than for the other two models (Fig. 5a,b). Moreover, the empirical distribution of correlation coefficients is better approximated by the FIC model than for the other two models (Fig. $5 c, d$ ).

Note that the decrease of the fitting of the empirical FC for small values of $G$ observed in simulations of BOLD signals (Fig. $3 b$ ) is more pronounced that the one obtained using the linear prediction of Q (Fig. 5a). This discrepancy arises from the distribution of functional correlation coefficients $r_{i j}$. Indeed, if the values are narrowly distributed, as for $G$ approaching zero for all models (Fig. 5c), the precise estimation of the correlation structure through stochastic simulations would require a large number of simulation steps, so that the estimation error of $r_{i j}$ becomes very small compared with the variance of the distribution of $r_{i j}$. Opposite to this, the analytic solution does not suffer from the sampling error and, thus, it correctly estimates the fine structure of $\mathbf{Q}$ with infinite resolution.

Furthermore, the linear approximation allows for an analytical estimation of the time-scale of the gating variables' fluctuations. Since the hemodynamic model used to simulate the BOLD fMRI signal can be seen as a low-pass filter that passes frequencies $<1 \mathrm{~Hz}$ (Robinson et al., 2006), only gating variables correlations at slow time scales are transmitted through the hemodynamic model. We thus calculated the power spectrum of fluctuations around the spontaneous state, by taking the Fourier transform of the equation governing the time evolution of fluctuations (see Materials and Methods; Fig. 5e). We found that in the FIC model the power of low-frequency $(<1 \mathrm{~Hz})$ fluctuations is maintained high for all values of $G$, whereas for the other two models slow fluctuations are expected for small values of $G$ only.

Altogether, the linear approximation shows that the FIC model better approximates the functional correlations and maintains the dynamics in a slow time-scale, so that the correlation structure of gating variables is seen through the hemodynamically filtered response.

\section{Feedback inhibition control increases the dynamical repertoire of the network evoked activity}

To examine the functional implications of FIC, we analyzed the response of the model network to exogenous stimuli, which are assumed to represent ideal task-related signals. We simulated 1000 different hypothetical task conditions by stimulating exogenously $10 \%$ of the brain areas, randomly selected. Stimulation was modeled by imposing an external input to the excitatory pools, i.e., for the set of stimulated excitatory pools we imposed $I_{\text {external }}=0.02$. The increased excitatory activity induces an evoked pattern of activity at the stationary state. We first calculated, for each model, the activity of cortical excitatory pool av- 
a
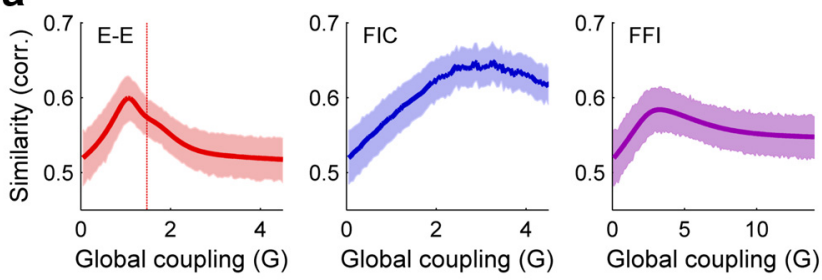

b

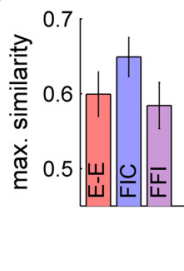

C

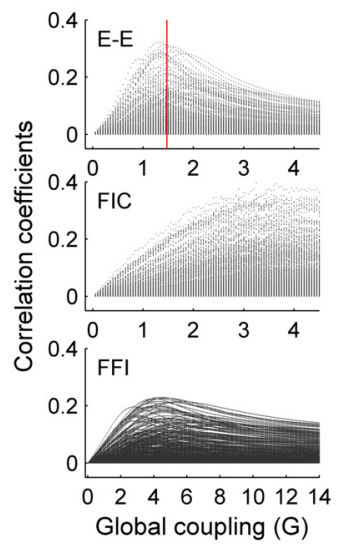

d

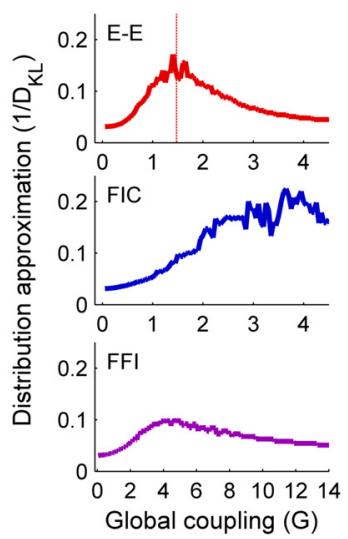

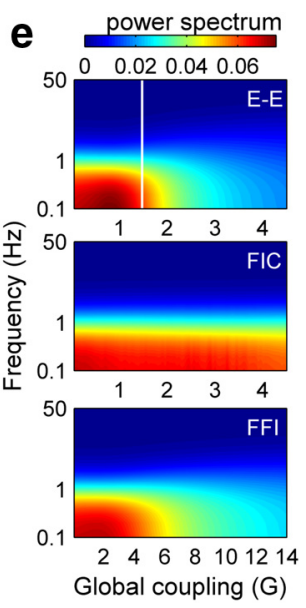

Figure 5. Linear fluctuations. $\boldsymbol{a}$, Similarity (correlation coefficient) between the empirical BOLD FC and the correlation structure of gating variables as obtained by the moments' method, for the three models. Shaded areas represent the $95 \%$ confidence interval of the similarity measure. In the FIC case, the same local feedback inhibition weights $\left(J_{i}\right)$ used in Figure $2 a, b$ were used here. $\boldsymbol{b}$, Comparison of the maximum similarity between the empirical BOLD FC and the correlation structure of gating variables for the three models. $c$, Correlation coefficients of all pairwise correlations between excitatory gating variables of the 66 cortical areas as a function of the global coupling $G$, for the three models. Top, The red line indicates the bifurcation of the E-E model. $\boldsymbol{d}$, Inverse of the Kullback-Leibler divergence $\left(D_{K L}\right)$ between the empirical distribution of correlation coefficients and the distribution of correlation coefficients between gating variables for each model. $\boldsymbol{e}$, Mean power spectrum of the excitatory gating variables as a function of the global coupling $G$, for the three different models. Top, The white line indicates the bifurcation of the E-E model.

eraged over all stimuli, for the corresponding optimal values of $G$ (Fig. 6a). Interesting, in both the E-E model and the FFI model, the activity is systematically much higher for some nodes, whether they are directly stimulated or not. These nodes are central nodes of the network, as revealed by their corresponding betweenness centrality (Fig. 6b), a network measure that quantifies the number of shortest paths that pass through a given node. Note that, as previously shown (Hagmann et al., 2008), central cortical areas are members of the default mode network (Fig. 6c).

We next characterized the information capacity of the largescale model by quantifying the size of the repertoire of different evoked patterns of both models. Indeed, a network that has few degrees of freedom, i.e., few different evoked patterns, has a low capability to transmit information. We thus hypothesized that the networks without FIC, due to their tendency to elevated the firing rate of central nodes in response to distinct stimuli, have less information capacity. We quantify this by calculating the entropy $H_{\text {evoked }}$ of binary evoked patterns (see Materials and Methods) to measure the extent of the repertoire of evoked activity. Binary patterns were constructed by imposing a threshold and setting the steady-state evoked activity of a given area to 0 or 1 whether it is below or above the threshold, respectively. The threshold was defined as $\theta . S D$, where SD is the standard deviation across all possible evoked responses of each model. Several values of the multiplier $\theta$ were tested. For each of the three large-scale models, we compared the entropy of evoked patterns to the maximum entropy by computing the reduction of entropy, defined as $\Delta H=\left(H_{\max }-H_{\text {evoked }}\right) / H_{\max }$, where the maximum entropy
$H_{\text {max }}$ is equal to $\log _{2}(n)$ for a set of $n$ possible binary patterns. Values of $\Delta H$ close to 0 indicate that the network has a large repertoire size, close to the maximum repertoire size, while values near 1 indicate a limited repertoire size. We found that, under FIC, the model has more entropy than the two other models (Fig. 6d), and, hence, it has a larger information capacity. The same result is obtained when stimuli are imposed to both the excitatory and the inhibitory population of the cortical areas (Fig. 6e).

\section{Feedback inhibition control increases the accuracy of the external stimulus encoding}

At last, we further asses the sensibility to external stimuli of the large-scale network. For this, we calculated the FI, which gives an upper bound to the accuracy that any population code can achieve (Abbott and Dayan, 1999). The FI takes into account the change of both the mean activity and the covariance with respect to a small variation of the stimulus strength to quantify the ability for stimulus discrimination (see Materials and Methods). Here, a "visual" stimulus was modeled by imposing an equal external input to both the right and the left LOCC. We used the moment's equations to precisely estimate the evoked stationary mean and covariance for varying stimulus intensity. The FI was calculated for each of the largescale models (Fig. $6 f$ ), where $G$ was set to the corresponding optimal value for each model. We found that the regulation of feedback inhibition increases the FI, thus increasing the accuracy of the stimulus encoding. Relaxing the FIC condition leads to a decrease of the FI because the central nodes are systematically activated in response to the stimulus, thus saturating the network response, although the FIC provides a graded response of the stimulated nodes with practically no activation of the other nodes (Fig. $6 g$ ).

\section{Discussion}

In this study, we investigated the impact of the local FIC on a large-scale model of the brain. We found several important and functionally relevant effects of the local FIC on both the spontaneous and the evoked patterns of activity of the large-scale model. First, we showed that the FIC significantly enhances the model's prediction of the fMRI human resting functional connectivity, even for single fMRI scanning sessions. Furthermore, we found that the optimal parameter space where the model best approximates the empirical FC is enlarged in the FIC condition. This is important since a common result of all previous resting-state models is that the optimal parameter space is confined at the edge of criticality (Honey et al., 2007, 2009; Ghosh et al., 2008; Deco et al., 2009, 2013a,b; Deco and Hughes, 2012) posing the problem of fine tuning for which self-organization rules remain unknown. Opposite to this, it has been shown that inhibitory synapses can rapidly adapt to changes in the local network activity to scale the activity in cortical circuits (Hartmann et al., 2008), thus providing a natural implementation of the FIC by biological synapses. In 

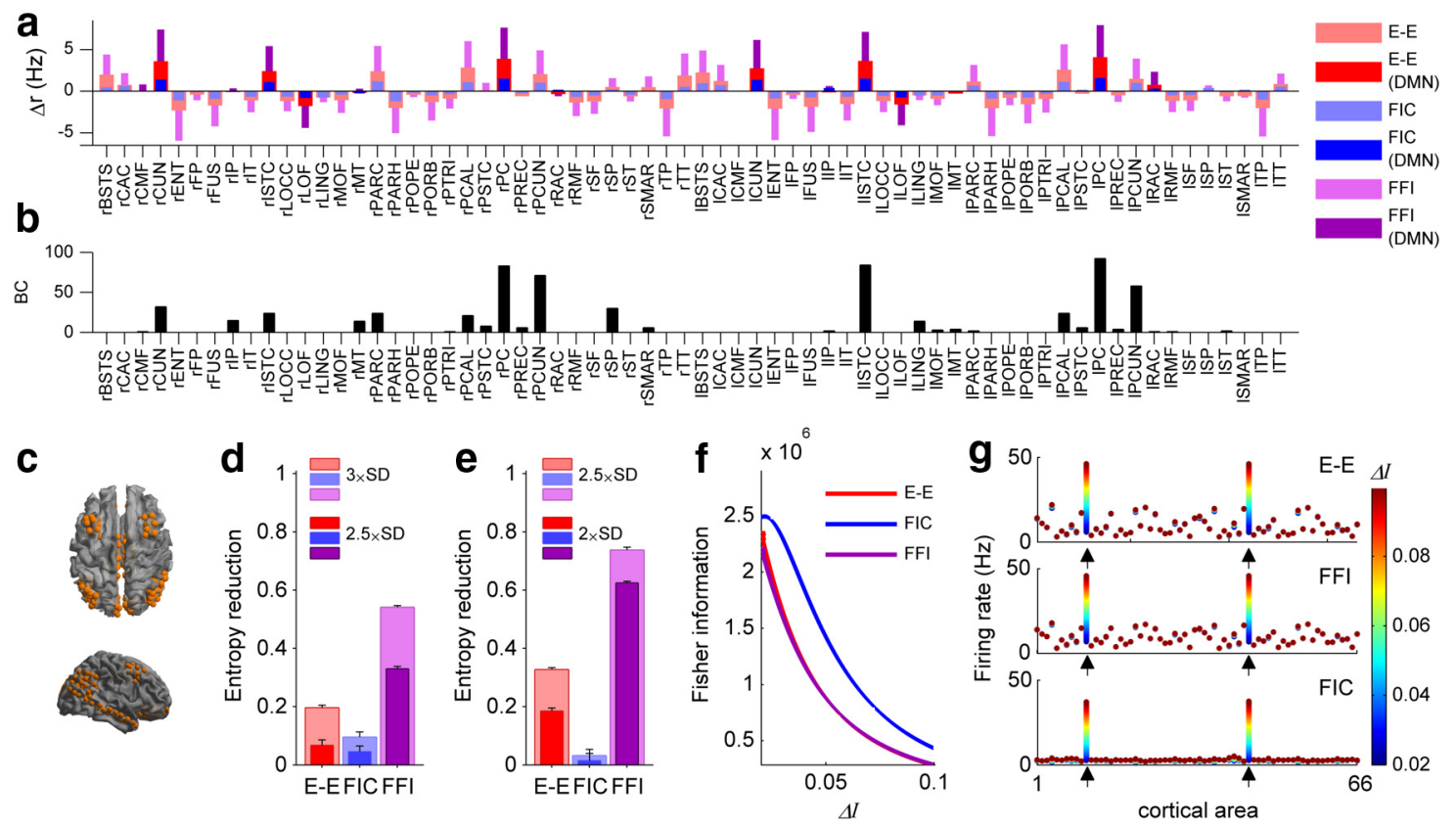

Figure 6. The effect of FIC on the evoked activity of the large-scale brain model. $\boldsymbol{a}$, Mean activity in each local brain area (with respect to the activity averaged over all brain areas) for each model obtained in response to 1000 different hypothetical task conditions. Tasks were simulated by imposing an external input $I_{\text {external }}=0.02$ to the excitatory population of $10 \%$ of the brain areas, randomly selected. Dark colors indicate the areas composing the default mode network. $\boldsymbol{b}$, Betweenness centrality (BC) of each brain area. BC was calculated from the anatomical connectivity matrix. It represents the number of shortest paths passing through a given node. $c$, The default mode network is composed of midline frontal and parietal areas, posterior inferior parietal lobule, and medial and lateral temporal lobe regions. $\boldsymbol{d}$, The entropy $H_{\text {evoked }}$ of evoked binary patterns was calculated for different activity thresholds, defined as $\theta$.SD, were SD is the standard deviation across all possible evoked responses. Bars indicate the reduction of entropy $\Delta H$, for the three different models, for different values of $\theta(\theta=2.5,3$, or 4$) . \Delta H$ was defined as $\Delta H=\left(H_{\max }-H_{\text {evoked }}\right) / H_{\text {max }}$ where $H_{\max }$ is the maximum entropy for the set of $n$ patterns, i.e., $H_{\max }=\log _{2}(n)$. Error bars indicate estimation errors $(50 \%)$ given by the quadratic extrapolation procedure, used for the entropy sampling bias correction. $\boldsymbol{e}$, Entropy reduction when external stimuli are imposed to both the excitatory and the inhibitory populations. The values of $\theta$ are lower than in (d) to avoid patterns with null entries. $\boldsymbol{f}$, The encoding accuracy of the models was calculated using the Fl. The FI was calculated for various stimulus intensities $(\Delta /)$. The stimulus was applied to the excitatory population of both right and left LOCC; i.e., $I_{\text {external }}=\Delta /$ for rLOCC and ILOCC, and $I_{\text {external }}=0$ for all other cortical areas. $\boldsymbol{g}$, Evoked response of the network for the E-E model (top), the FFI model (middle), and for the FIC model (bottom). The colors indicate the intensity $(\Delta /)$ of the applied stimulus. The arrows indicate the stimulated nodes.

this view the local FIC may provide a homeostatic mechanism that generates the observed resting-state FC. Nevertheless, in the present study we have regulated the inhibitory-to-excitatory coupling, but manipulating other local weights to adjust the ratio between excitation and inhibition in the large-scale model may lead to similar results and would provide a local decorrelation mechanism, providing that excitation and inhibition balance each other (Renart et al., 2010).

Nevertheless, note that the agreement between the model's FC and the empirical FC is not perfect. This imperfect fitting is expected principally due to the missed of connections of the diffusion tensor/spectrum imaging (DTI/DSI) tractography, used here to estimate the anatomical connectivity that constrains the large-scale connectivity of the model. Indeed, precise prediction of the FC strongly depends on the quality of the estimated structural connectivity. However, interhemispherical connections are not well captured by the DTI/DSI tractography (Hagmann et al., 2008) and the anatomical matrix used here did not include subcortical routes that are known to play an important role in shaping the spontaneous activity of the brain (Robinson et al., 2001; Freyer et al., 2011). Estimation of subcortical connections is a challenging issue because DTI systematically leads to more connections for proximal regions than distal ones, and thus, subcortical structures are particularly subject to this bias (Jones, 2008). Moreover, neural structure is different in the cortex and subcortical nuclei, for example, the thalamus is composed of GABAergic interneurons in the reticular nucleus of the thalamus and thalamocortical relay neurons in relay nuclei, for which relative number of neurons is unknown (Izhikevich and Edelman, 2008), and thus, how both the cortex and the subcortical nuclei can be described in a parsimonious model is an open question.

In addition, we have made several simplifying assumptions that may impose an upper bound to the agreement between the model predictions and the empirical observations. Specifically, in this study we consider that all connections between brain areas are instantaneous, thus neglecting the effects of conduction delays which are likely to shape the brain dynamics, giving rise to complex spatiotemporal patterns, oscillations, multistability, and chaos (Roxin et al., 2005; Ghosh et al., 2008; Deco et al., 2009). Indeed, a recent MEG study found robust frequency-specific lagged coherence between interhemispheric functional connections in the dorsal attention network, or between the dorsal attention and other networks (e.g., visual and somatomotor; Marzetti et al., 2013). Considering the delays would make more complex the model and add a new parameter to explore: the propagation velocity.

In this work, we assumed that functional connectivity is a stationary process that arises from the interplay between the underlying anatomical connectivity structure and the neural dynamics on the stationary regime. This assumption is commonly made by the current resting-state models that seek to link the structural and the functional connectivity (Honey et al., 2007, 2009; Ghosh et al., 2008; Deco et al., 2009, 2013a,b; Deco and Hughes, 2012). However, recent studies have demonstrated that the correlations among brain regions evolve over time (Chang and Glover, 2010; Kiviniemi et al., 2011; de Pasquale et al., 2012; Hutchison et al., 2013; Allen et al., 2014). Because nonstationarities are likely to substantially contribute to the resting-state activity (Messé et al., 2014), it is thus crucial to analyze and model the 
time-varying behavior of the functional connectivity. How nonstationary functional connectivity emerges in the brain network remains an open question that needs further investigation.

In the present study, we assumed that the spontaneous activity throughout the cortex is low. This is supported by early recordings of cortical activity in vivo (Burns and Webb, 1976; Softky and Koch, 1993; Wilson et al., 1994), as well as recent experiments in mammalian neocortex using diverse recording techniques, such as whole-cell patch-clamp, sharp-electrode recordings and $\mathrm{Ca}^{2+}$-imaging, in different brain states (awake or under anesthesia), indicating that the spontaneous activity of pyramidal neurons in sensory cortices ranges between $1-5 \mathrm{~Hz}$, with further laminar differences (Sakata and Harris, 2012; for review, see Barth and Poulet, 2012). Nevertheless, more investigation comparing the spontaneous activity of different sensory and association cortices is needed.

By studying the large-scale patterns evoked by external inputs, we showed that, if the FIC constraint is relaxed, external stimulation of the brain model leads to a systematic large activation of cortical areas that are components of the default mode network. This is unlikely to occur in the real brain, because only few experimental conditions leading to activation of the default mode network have been reported. Indeed, a myriad of reports shows that the default network is deactivated during attentionally demanding and goal-directed tasks (Shulman et al., 1997; Raichle et al., 2001; Fox and Raichle, 2007; Thomason et al., 2008; Christoff et al., 2009). Nevertheless, the default mode network is activated by cognitive processes that are internally driven, such as, memory retrieval (Sestieri et al., 2011), internal mentation (Mason et al., 2007; Bar, 2009), and self-reference (Gusnard and Raichle, 2001; D'Argembeau et al., 2005). However, these internal processes are more likely to be initiated spontaneously, possibly due to spontaneous fluctuations in the cortex, as recently proposed in the context of self-initiated movements (Schurger et al., 2012), and thus, they may not be well modeled by imposing external inputs to some cortical areas. Altogether, we believe that the systematic activation of cortical areas belonging to the default mode network, observed in the evoked responses of the brain models without FIC, is not consistent either with current experimental data or with current assumptions about the generation of internal mental states. Here we showed that regulating the local level of feedback inhibition in the brain has an important role at the global level, because it attenuates the response of cortical areas in the default mode network.

A further effect of local FIC on large-scale brain patterns is to increase the entropy of the evoked activity patterns, thus increasing the dynamic repertoire of the network. Hence, the local FIC enhances the information capacity of the global network, i.e., it increases the ability of the network to map different inputs into distinguishable network outputs. This result complements previously reported evidence showing that neural systems tend to maximize the entropy at the level of single neurons (Tsubo et al., 2012) and neural populations (Shew et al., 2011). It has been shown that when excitation is sufficiently restrained by inhibition in local cortical networks, the information capacity of cortical circuits is increased (Shew et al., 2011; Deco and Hughes, 2012). Furthermore, we showed that regulation of the feedback inhibition enhances the stimulus discriminability, i.e., it increases the sensitivity of the network to a small variation of the stimulus. Hence, our results indicate that the control of the excitation-inhibition ratio at the local level has important implications for information transmission at the large-scale brain level. Moreover, because the feedback inhibition acts as an active mechanism for reducing pairwise correlations in local net- works (Tetzlaff et al., 2012; Fig. 1), it improves the encoding and readout within local circuits.

In summary, we had identified several effects of local regulation of feedback inhibition on brain dynamics at the large scale: it changes the characteristics of the emergent resting and evoked activity in a crucial way. Regulating the local excitation-inhibition ratio provides a better and more robust prediction of human empirical resting state connectivity, together with more realistic responses to external inputs and higher information capacity and encoding accuracy.

\section{References}

Abbott LF, Dayan P (1999) The effect of correlated variability on the accuracy of a population code. Neural Comput 11:91-101. CrossRef Medline

Achard S, Salvador R, Whitcher B, Suckling J, Bullmore E (2006) A resilient, low-frequency, small-world human brain functional network with highly connected association cortical hubs. J Neurosci 26:63-72. CrossRef Medline

Allen EA, Damaraju E, Plis SM, Erhardt EB, Eichele T, Calhoun VD (2014) Tracking whole-brain connectivity dynamics in the resting state. Cereb Cortex 24:663-676. CrossRef Medline

Bar M (2009) The proactive brain: memory for predictions. Philos Trans R Soc Lond B Biol Sci 364:1235-1243. CrossRef Medline

Barth AL, Poulet JF (2012) Experimental evidence for sparse firing in the neocortex. Trends Neurosci 35:345-355. CrossRef Medline

Bassett DS, Meyer-Lindenberg A, Achard S, Duke T, Bullmore E (2006) Adaptive reconfiguration of fractal small-world human brain functional networks. Proc Natl Acad Sci U S A 103:19518-19523. CrossRef Medline

Biswal B, Yetkin FZ, Haughton VM, Hyde JS (1995) Functional connectivity in the motor cortex of resting human brain using echo-planar MRI. Magn Reson Med 34:537-541. CrossRef Medline

Brookes MJ, Woolrich M, Luckhoo H, Price D, Hale JR, Stephenson MC, Barnes GR, Smith SM, Morris PG (2011) Investigating the electrophysiological basis of resting state networks using magnetoencephalography. Proc Natl Acad Sci U S A 108:16783-16788. CrossRef Medline

Burns BD, Webb AC (1976) The spontaneous activity of neurons in the cat's cerebral cortex. Proc R Soc Lond Biol 194:211-223. CrossRef Medline

Chang C, Glover GH (2010) Time-frequency dynamics of resting-state brain connectivity measured with fMRI. Neuroimage 50:81-98. CrossRef Medline

Christoff K, Gordon AM, Smallwood J, Smith R, Schooler JW (2009) Experience sampling during fMRI reveals default network and executive system contributions to mind wandering. Proc Natl Acad Sci U S A 106: 8719-8724. CrossRef Medline

D’Argembeau A, Collette F, Van der Linden M, Laureys S, Del Fiore G, Degueldre C, Luxen A, Salmon E (2005) Self-referential reflective activity and its relationship with rest: a PET study. Neuroimage 25:616-624. CrossRef Medline

Deco G, Hugues E (2012) Balanced input allows optimal encoding in a stochastic binary neural network model: an analytical study. PLoS ONE 7:e30723. CrossRef Medline

Deco G, Jirsa VK (2012) Ongoing cortical activity at rest: criticality, multistability, and ghost attractors. J Neurosci 32:3366-3375. CrossRef Medline

Deco G, Jirsa V, McIntosh AR, Sporns O, Kötter R (2009) Key role of coupling, delay, and noise in resting brain fluctuations. Proc Natl Acad Sci U S A 106:10302-10307. CrossRef Medline

Deco G, Ponce-Alvarez A, Mantini D, Romani GL, Hagmann P, Corbetta M (2013a) Resting-state functional connectivity emerges from structurally and dynamically shaped slow linear fluctuations. J Neurosci 33:1123911252. CrossRef Medline

Deco G, Jirsa VK, McIntosh AR (2013b) Resting brains never rest: computational insights into potential cognitive architectures. Trends Neurosci 36:268-274. CrossRef Medline

de Pasquale F, Della Penna S, Snyder AZ, Marzetti L, Pizzella V, Romani GL, Corbetta M (2012) A cortical core for dynamic integration of functional networks in the resting human brain. Neuron 74:753-764. CrossRef Medline

Doucet G, Naveau M, Petit L, Delcroix N, Zago L, Crivello F, Jobard G, Tzourio-Mazoyer N, Mazoyer B, Mellet E, Joliot M (2011) Brain activity at rest: a multiscale hierarchical functional organization. J Neurophysiol 105:2753-2763. CrossRef Medline

Ecker AS, Berens P, Keliris GA, Bethge M, Logothetis NK, Tolias AS (2010) 
Decorrelated neuronal firing in cortical microcircuits. Science 327:584587. CrossRef Medline

Fox MD, Raichle ME (2007) Spontaneous fluctuations in brain activity observed with functional magnetic resonance imaging. Nat Rev Neurosci 8:700-711. CrossRef Medline

Fransson P (2005) Spontaneous low-frequency BOLD signal fluctuations: an fMRI investigation of the resting-state default mode of brain function hypothesis. Hum Brain Mapp 26:15-29. CrossRef Medline

Freyer F, Roberts JA, Becker R, Robinson PA, Ritter P, Breakspear M (2011) Biophysical mechanisms of multistability in resting-state cortical rhythms. J Neurosci 31:6353-6361. CrossRef Medline

Friston KJ, Harrison L, Penny W (2003) Dynamic causal modelling. Neuroimage 19:1273-1302. CrossRef Medline

Ghosh A, Rho Y, McIntosh AR, Kötter R, Jirsa VK (2008) Noise during rest enables the exploration of the brain's dynamic repertoire. PLoS Comput Biol 4:e1000196. CrossRef Medline

Greicius MD, Krasnow B, Reiss AL, Menon V (2003) Functional connectivity in the resting brain: a network analysis of the default mode hypothesis. Proc Natl Acad Sci U S A 100:253-258. CrossRef Medline

Gusnard DA, Raichle ME (2001) Searching for a baseline: functional imaging and the resting human brain. Nat Rev Neurosci 2:685-694. CrossRef Medline

Hacker CD, Laumann TO, Szrama NP, Baldassarre A, Snyder AZ, Leuthardt EC, Corbetta M (2013) Resting state network estimation in individual subjects. Neuroimage 82:616-633. CrossRef Medline

Hagmann P, Cammoun L, Gigandet X, Meuli R, Honey CJ, Wedeen VJ, Sporns O (2008) Mapping the structural core of human cerebral cortex. PLoS Biol 6:e159. CrossRef Medline

Hartmann K, Bruehl C, Golovko T, Draguhn A (2008) Fast homeostatic plasticity of inhibition via activity-dependent vesicular filling. PLoS ONE 3:e2979. CrossRef Medline

Honey CJ, Kötter R, Breakspear M, Sporns O (2007) Network structure of cerebral cortex shapes functional connectivity on multiple time scales. Proc Natl Acad Sci U S A 104:10240-10245. CrossRef Medline

Honey CJ, Sporns O, Cammoun L, Gigandet X, Thiran JP, Meuli R, Hagmann P (2009) Predicting human resting-state functional connectivity from structural connectivity. Proc Natl Acad Sci U S A 106:2035-2040. CrossRef Medline

Hutchison RM, Womelsdorf T, Gati JS, Everling S, Menon RS (2013) Resting-state networks show dynamic functional connectivity in awake humans and anesthetized macaques. Hum Brain Mapp 34:2154-2177. CrossRef Medline

Izhikevich EM, Edelman GM (2008) Large-scale model of mammalian thalamocortical systems. Proc Natl Acad Sci U S A 105:3593-3598. CrossRef Medline

Jones DK (2008) Studying connections in the living human brain with diffusion MRI. Cortex 44:936-952. CrossRef Medline

Kiviniemi V, Vire T, Remes J, Elseoud AA, Starck T, Tervonen O, Nikkinen J (2011) A sliding time-window ICA reveals spatial variability of the default mode network in time. Brain Connectivity 1:339-347. CrossRef Medline

Liu Z, Fukunaga M, de Zwart JA, Duyn JH (2010) Large-scale spontaneous fluctuations and correlations in brain electrical activity observed with magnetoencephalography. Neuroimage 51:102-111. CrossRef Medline

Luckhoo H, Hale JR, Stokes MG, Nobre AC, Morris PG, Brookes MJ, Woolrich MW (2012) Inferring task-related networks using independent component analysis in magnetoencephalography. Neuroimage 62:530541. CrossRef Medline

Mantini D, Perrucci MG, Del Gratta C, Romani GL, Corbetta M (2007) Electrophysiological signatures of resting state networks in the human brain. Proc Natl Acad Sci U S A 104:13170-13175. CrossRef Medline

Mantini D, Corbetta M, Romani GL, Orban GA, Vanduffel W (2013) Evolutionarily novel functional networks in the human brain? J Neurosci 33:3259-3275. CrossRef Medline

Marzetti L, Della Penna S, Snyder AZ, Pizzella V, Nolte G, de Pasquale F, Romani GL, Corbetta M (2013) Frequency specific interactions of MEG resting state activity within and across brain networks as revealed by the multivariate interaction measure. Neuroimage 79:172-183. CrossRef Medline

Mason MF, Norton MI, Van Horn JD, Wegner DM, Grafton ST, Macrae CN (2007) Wandering minds: the default network and stimulusindependent thought. Science 315:393-395. CrossRef Medline

Messé A, Rudrauf D, Benali H, Marrelec G (2014) Relating structure and function in the human brain: relative contributions of anatomy, stationary dynamics, and non-stationarities. PLoS Comput Biol 10:e1003530. CrossRef Medline

Power JD, Cohen AL, Nelson SM, Wig GS, Barnes KA, Church JA, Vogel AC, Laumann TO, Miezin FM, Schlaggar BL, Petersen SE (2011) Functional network organization of the human brain. Neuron 72:665-678. CrossRef Medline

Raichle ME, MacLeod AM, Snyder AZ, Powers WJ, Gusnard DA, Shulman GL (2001) A default mode of brain function. Proc Natl Acad Sci U S A 98:676-682. CrossRef Medline

Renart A, de la Rocha J, Bartho P, Hollender L, Parga N, Reyes A, Harris KD (2010) The asynchronous state in cortical circuits. Science 327:587-590. CrossRef Medline

Robinson PA, Rennie CJ, Wright JJ, Bahramali H, Gordon E, Rowe DL (2001) Prediction of electroencephalographic spectra from neurophysiology. Phys Rev E Stat Nonlin Soft Matter Phys 63:021903. CrossRef Medline

Robinson PA, Drysdale PM, Van der Merwe H, Kyriakou E, Rigozzi MK, Germanoska B, Rennie CJ (2006) BOLD responses to stimuli: dependence on frequency, stimulus form, amplitude, and repetition rate. Neuroimage 31:585-599. CrossRef Medline

Roxin A, Brunel N, Hansel D (2005) Role of delays in shaping spatiotemporal dynamics of neuronal activity in large networks. Phys Rev Lett 94: 238103. CrossRef Medline

Sakata S, Harris KD (2012) Laminar-dependent effects of cortical state on auditory cortical spontaneous activity. Front Neural Circuits 6:109. CrossRef Medline

Schurger A, Sitt JD, Dehaene S (2012) An accumulator model for spontaneous neural activity prior to self-initiated movement. Proc Natl Acad Sci U S A 109:E2904-2913. CrossRef Medline

Sestieri C, Corbetta M, Romani GL, Shulman GL (2011) Episodic memory retrieval, parietal cortex, and the default mode network: functional and topographic analyses. J Neurosci 31:4407-4420. CrossRef Medline

Shew WL, Yang H, Yu S, Roy R, Plenz D (2011) Information capacity and transmission are maximized in balanced cortical networks with neuronal avalanches. J Neurosci 31:55-63. CrossRef Medline

Shulman GL, Fiez JA, Corbetta M, Buckner RL, Miezin FM, Raichle ME, Petersen SE (1997) Common blood flow changes across visual tasks: II. Decreases in cerebral cortex. J Cogn Neurosci 9:648-663. CrossRef Medline

Softky WR, Koch C (1993) The highly irregular firing of cortical cells is inconsistent with temporal integration of random EPSPs. J Neurosci 13: 334-350. Medline

Sui J, Adali T, Pearlson GD, Calhoun VD (2009) An ICA-based method for the identification of optimal FMRI features and components using combined group-discriminative techniques. Neuroimage 46:73-86. CrossRef Medline

Tetzlaff T, Helias M, Einevoll GT, Diesmann M (2012) Decorrelation of neural-network activity by inhibitory feedback. PLoS Comput Biol 8:e1002596. CrossRef Medline

Thomason ME, Chang CE, Glover GH, Gabrieli JD, Greicius MD, Gotlib IH (2008) Default-mode function and task-induced deactivation have overlapping brain. Neuroimage 41:1493-1503. CrossRef Medline

Treves A, Panzeri S (1995) The upward bias in measures of information derived from limited data samples. Neural Comput 7:399-407. CrossRef

Tsubo Y, Isomura Y, Fukai T (2012) Power-law inter-spike interval distributions infer a conditional maximization of entropy in cortical neurons. PLoS Comput Biol 8:e1002461. CrossRef Medline

van Vreeswijk C, Sompolinsky H (1996) Chaos in neuronal networks with balanced excitatory and inhibitory activity. Science 274:1724-1726. CrossRef Medline

Wilson FA, Scalaidhe SP, Goldman-Rakic PS (1994) Functional synergism between putative gamma-aminobutyrate-containing neurons and pyramidal neurons in prefrontal cortex. Proc Natl Acad Sci U S A 91:40094013. CrossRef Medline

Wong KF, Wang XJ (2006) A recurrent network mechanism of time integration in perceptual decisions. J Neurosci 26:1314-1328. CrossRef Medline

Yeo BT, Krienen FM, Sepulcre J, Sabuncu MR, Lashkari D, Hollinshead M, Roffman JL, Smoller JW, Zöllei L, Polimeni JR, Fischl B, Liu H, Buckner RL (2011) The organization of the human cerebral cortex estimated by functional connectivity. J Neurophysiol 106:1125-1165. CrossRef Medline 